\title{
Desynchronization of pulse-coupled oscillators with delayed excitatory coupling 1
}

\author{
Wei $\mathrm{Wu}^{2}$, Tianping Chen 3
}

\begin{abstract}
Collective behavior of pulse-coupled oscillators has been investigated widely. As an example of pulse-coupled networks, fireflies display many kinds of flashing patterns. Mirollo and Strogatz (1990) proposed a pulse-coupled oscillator model to explain the synchronization of South East Asian fireflies (Pteroptyx malaccae). However, transmission delays were not considered in their model. In fact, the presence of transmission delays can lead to desychronization. In this paper, pulse-coupled oscillator networks with delayed excitatory coupling are studied. Our main result is that under reasonable assumptions, pulse-coupled oscillator networks with delayed excitatory coupling can not achieve complete synchronization, which can explain why another species of fireflies (Photinus pyralis) rarely synchronizes flashing. Finally, two numerical simulations are given. In the first simulation, we illustrate that even if all the initial phases are very close to each other, there could still be big variations in the times to process the pulses in the pipeline. It implies that asymptotical synchronization typically also cannot be achieved. In the second simulation, we exhibit a phenomenon of clustering synchronization.
\end{abstract}

PACS numbers: 87.10.+e, 05.45.+b

Key words: Synchronization; Desynchronization; Pulse-coupled oscillators

\footnotetext{
${ }^{1}$ This work is supported by National Science Foundation of China 60374018, 60574044 and 973 Program 2005 CB523306.

${ }^{2} \mathrm{He}$ is with Lab. of Nonlinear Mathematics Science, Institute of Mathematics, Fudan University, Shanghai, 200433, P. R. China Email: wuweifd@vip.sina.com

${ }^{3} \mathrm{He}$ is with Lab. of Nonlinear Mathematics Science, Institute of Mathematics, Fudan University, Shanghai, 200433, P. R. China.

Corresponding author: Tianping Chen. Email: tchen@fudan.edu.cn;
} 


\section{Introduction}

Fireflies provide one of the most spectacular examples of synchronization in nature [1, 2, 3, 4. Through observation, people discovered that there are several synchronization patterns in different species of fireflies. Some species, such as Pteroptyx malaccae, flash rhythmically in perfect unison [1. Some other species, such as Photinus pyralis, usually show clustering synchrony or wave sweeping synchrony, instead of complete synchrony [2].

Many kinds of biological models have been studied for flashing behavior of fireflies. A pioneering work for synchronization of fireflies has been reported by Buck in [2]. He investigated a variety of firefly species including Pteroptyx malaccae, Pteroptyx cribellata and Photinus pyralis. He suggested two kinds of flash models, phase-advance entrainment model and phase-delay entrainment model. In the phase advance model displayed in Fig.1(a), an excitation level of pace-maker in the firefly brain is enhanced by an external light stimulation. Each time excitation reaches a threshold level, a neural signal is transmitted into the light organ in the abdomen and then a flashing light is produced. An example of this model is Photinus pyralis, the whole group of the species rarely synchronizes flashing [2]. Instead, "wave", "chain" or "sweeping" synchrony has been reported in some species with this phase advance model. A cluster of male fireflies flash and then the flash of light is dispersed into neighbor males. In the phase delay model displayed in Fig.1(b), an excitation potential of pace-maker is reset to the basal level by a light stimulation and the potential increase is restarted to reach the threshold level for flashing. Pteroptyx cribellata is known as an example of the phase delay model and it usually shows a complete synchronization.

(a)

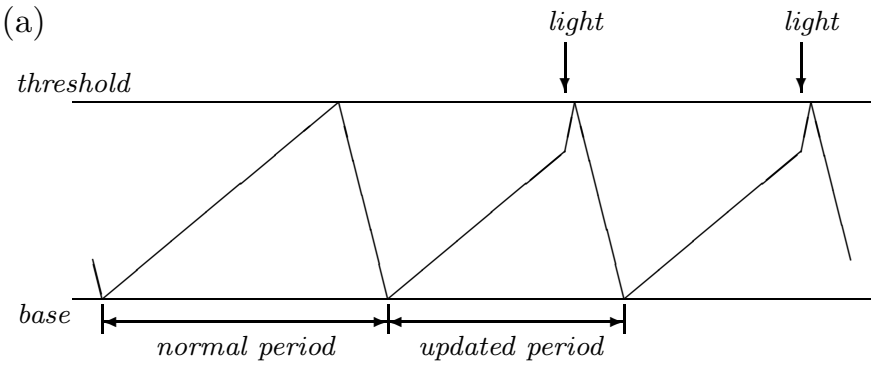

(b)

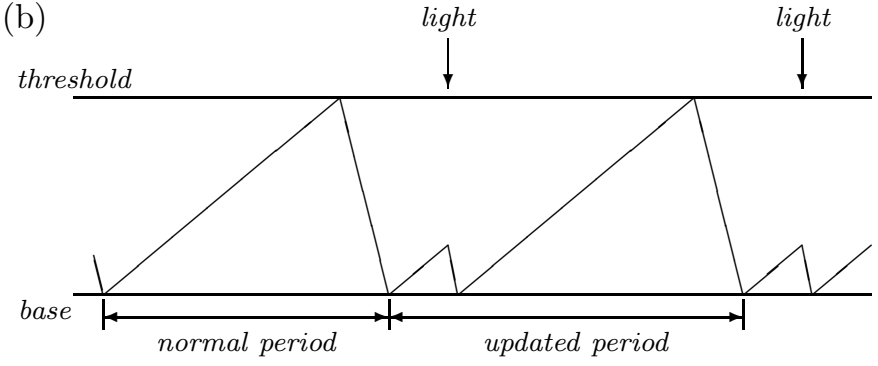

Fig.1: (a) Phase advance model (b) Phase delay model. (Reprinted from [2])

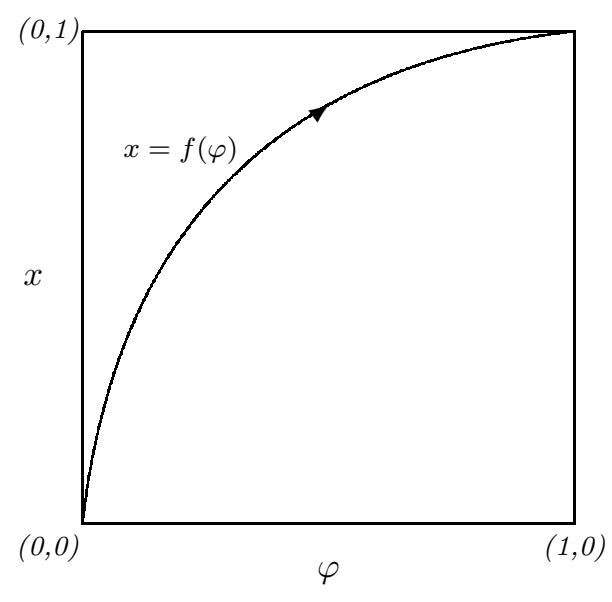

Fig.2: Graph of the function $f$. The time-course of the oscillator is given by $x=f(\varphi)$. 
Mirollo and Strogatz (1990) argued that Buck's model is not appropriate for synchronization because he assumed a linearly increasing potential toward threshold as shown in Fig.1. Inspired by Peskin's model for self-synchronization of the cardiac pacemaker [5], Mirollo and Strogatz proposed a pulse-coupled oscillator model with undelayed excitatory coupling to explain the synchronization of huge congregations of South East Asian fireflies (Pteroptyx malaccae) [6]. This model is a network of $N$ pulse-coupled oscillators. Each oscillator is characterized by a state variable $x_{i}$ which is assumed to increase toward a threshold at $x_{i}=1$ according to $x_{i}=f\left(\varphi_{i}\right)$, where $f:[0,1] \rightarrow[0,1]$ is smooth, monotonic increasing, and concave down, i.e., $f^{\prime}>0$ and $f^{\prime \prime}<0$. Here $\varphi_{i} \in[0,1]$ is a phase variable such that (i) $\mathrm{d} \varphi_{i} / \mathrm{d} t=1 / T$, where $T$ is the cycle period, (ii) $\varphi_{i}=0$ when the $i$ th oscillator is at its lowest state $x_{i}=0$, and (iii) $\varphi_{i}=1$ at the end of the cycle when the $i$ th oscillator reaches the threshold $x_{i}=1$. Therefore $f$ satisfies $f(0)=0$, $f(1)=1$. Fig. 2 shows the graph of a typical $f$. When $x_{i}$ reaches the threshold, the $i$ th oscillator "fires" and $x_{i}$ jumps back instantly to zero, after which the cycle repeats. The oscillators are assumed to interact by a simple form of pulse-coupling: when a given oscillator fires, it pulls all the other oscillators up by an amount $\varepsilon$, or pulls them up to firing, whichever is less. That is,

$$
x_{i}(t)=1 \Rightarrow x_{j}\left(t^{+}\right)=\min \left(1, x_{j}(t)+\varepsilon\right), \quad \forall j \neq i .
$$

The main result in [6] is that for all $N$ and for almost all initial conditions, the system eventually becomes completely synchronized. Here, the concept of complete synchronization is defined as follows: if there exists a $t_{0} \geq 0$ such that

$$
x_{i}(t)=x_{j}(t), \quad \text { for all } t \geq t_{0} \text { and all } i \neq j
$$

then the pulse-coupled oscillator network is said to be completely synchronized or, for simplicity, synchronized.

Since Mirollo and Strogatz's model was introduced, many results on pulse-coupled networks with undelayed coupling, including undelayed excitatory coupling and undelayed inhibitory coupling, have been obtained [7, 8, 9, 10, 11, 12. However, reaction and transmission delays are unavoidable in real biological systems. For example, normally the transmission delays of most fireflies from sensors to motor actions of flashing are around 200ms (see [2]). In fact, transmission delays influence the performance of synchronization for both excitatory and inhibitory coupling [13, 14, 15]. In particular, it has been shown that for excitatory coupling, the presence of transmission delays can lead to desychronization [13, 14, 16, 17]. To the best of our knowledge, this desynchronization was proved only for the case of two pulse-coupled oscillators, while for the case of $N>2$ pulse-coupled oscillators, it was revealed only in simulations. 
In this paper, pulse-coupled oscillator networks with delayed excitatory coupling are studied. We retain two of Mirollo and Strogatz's assumptions: the oscillators have identical dynamics, and all oscillators are coupled to all the others. Our main contribution is that we prove that $N \geq 2$ pulse-coupled oscillators with delayed excitatory coupling can not achieve complete synchronization. This result can explain why Photinus pyralis rarely synchronizes flashing, which is known as an example of pulse-coupled oscillator networks with delayed excitatory coupling [2]. Finally, two numerical simulations are given. In Simulation 1, we illustrate that even if all the initial phases are very close to each other, there could still be big variations in the times to process the pulses in the pipeline. It implies that asymptotical synchronization typically also cannot be achieved. In Simulation 2, we exhibit a phenomenon of clustering synchronization.

The rest of the paper is organized as follows: In Section 2, we describe the network model. In Section 3, some definitions and notations are given. In Section 4, some lemmas are proved. In Section 5, we prove the main theorem. Two numerical simulations are given in Section 6 . We conclude the paper in Section 7.

\section{Model}

The network consists of $N \geq 2$ pulse-coupled oscillators with delayed excitatory coupling. As in [6], the oscillators have identical dynamics, and all oscillators are coupled to all the others. Each oscillator is characterized by a state variable $x_{i}$ which is assumed to increase toward a threshold at $x_{i}=1$ according to $x_{i}=f\left(\varphi_{i}\right)$, where $f:[0,1] \rightarrow[0,1]$ is smooth, monotonic increasing, and concave down, i.e., $f^{\prime}>0$ and $f^{\prime \prime}<0$. Here $\varphi_{i} \in[0,1]$ is a phase variable such that (i) $\mathrm{d} \varphi_{i} / \mathrm{d} t=1 / T$, where $T$ is the cycle period, (ii) $\varphi_{i}=0$ when the $i$ th oscillator is at its lowest state $x_{i}=0$, and (iii) $\varphi_{i}=1$ at the end of the cycle when the $i$ th oscillator reaches the threshold $x_{i}=1$. When $x_{i}$ reaches the threshold, the $i$ th oscillator fires and $x_{i}$ jumps back instantly to zero, after which the cycle repeats. That is,

$$
x_{i}(t)=1 \Rightarrow x_{i}\left(t^{+}\right)=0 .
$$

The new features are that, (i) in order to make the discussion a little easier, we let $T=1$,

i.e., $\mathrm{d} \varphi_{i} / \mathrm{d} t=1$, and (ii) the transmission delay $\tau$ is introduced into the model. Because of the presence of the transmission delay, the oscillators interact by a new form: when a given oscillator fires at time $t$, it emits a spike; after a transmission delay $\tau$, the spike reaches all the other oscillators at time $t+\tau$ and pulls them up by an amount $\varepsilon$, or pulls them up to firing, whichever 
is less. That is,

$$
x_{i}(t)=1 \Rightarrow x_{j}(t+\tau)=\min \left(1, x_{j}\left((t+\tau)^{-}\right)+\varepsilon\right), \quad \forall j \neq i,
$$

instead of (1). Of course, if there are $m$ oscillators firing simultaneously, then the state variables $x$ of the $m$ oscillators are increased by an amount $(m-1) \varepsilon$ and the state variables $x$ of all the other oscillators are increased by an amount $m \varepsilon$. By (3) and (4), it is clear that if a spike reaches the $i$ th oscillator just at the moment that the $i$ th oscillator reaches the threshold $x_{i}=1$, then the state variable $x_{i}$ changes as follows:

$$
x_{i}(t)=\min \left(1, x_{i}\left(t^{-}\right)+\varepsilon\right)=\min (1,1+\varepsilon)=1 \Rightarrow x_{i}\left(t^{+}\right)=0 .
$$

In addition, we make the following two assumptions:

(A1) The system is started at time $t=0$ with a set of initial states $0<x_{i}(0) \leq 1$, and there are no firings in time $[-\tau, 0)$.

(A2) The transmission delay $\tau$ and the coupling strength $\varepsilon$ satisfy $f(2 \tau)+N \varepsilon<1$.

The assumption (A1) about the initial conditions is quite common for pulse-coupled networks with transmission delays [13, 14, 16, 17]. The time $t=0$ can be regarded as the moment at which fireflies assemble and begin to flash. Since $x_{i}$ jumps back instantly to 0 when it reaches the threshold 1 , the state values 0 and 1 may be considered to be the same state. Therefore, we assume that the initial states satisfy $0<x_{i}(0) \leq 1$ instead of $0 \leq x_{i}(0) \leq 1$.

The assumption (A2) means that both $\tau$ and $\varepsilon$ are relatively small. It is reasonable to fireflies with the phase advance entrainment model, especially to Photinus pyralis. The transmission delays of most fireflies from sensors to motor actions of flashing are around 200ms, while the regular endogenous period of Photinus pyralis is almost 6000ms (see [2]). Normally, excitatory activations generate frequent spikes in a short period of time, because it can shorten the period of oscillator by increasing the potential level (see [18]). It means that strong excitations result in frequent flashing of light, which will exhaust out the energy of the insect. Thus, a relatively small coupling strength for excitatory action is used in many species of fireflies, which follow the phase advance model. In the following sections, one can see that under this assumption, the number of case distinctions will be reduced.

With the monotonicity of $f$, the state variable $x_{i}$ and the phase variable $\varphi_{i}$ are one-to-one correspondence. Therefore, the synchronization of the state variables $x_{1}, \ldots, x_{N}$ is equivalent to the synchronization of the phase variables $\varphi_{1}, \ldots, \varphi_{N}$. In the following, instead of investigating $x_{i}$, we investigate dynamical behaviors of $\varphi_{i}$ directly. 
From the above description, the phase variable processes the following properties.

Proposition 1 The phase variable $\varphi_{i}$ satisfies:

(a) If $m$ spikes reach the ith oscillator at time $t$, then $\varphi_{i}(t)=f^{-1}\left(\min \left[1, f\left(\varphi_{i}\left(t^{-}\right)\right)+m \varepsilon\right]\right)$;

If no spikes reach the ith oscillator at time $t$, then $\varphi_{i}(t)=\varphi_{i}\left(t^{-}\right)$.

(b) If $\varphi_{i}(t)=1$, then $\varphi_{i}\left(t^{+}\right)=0$;

If $\varphi_{i}(t)<1$, then $\varphi_{i}\left(t^{+}\right)=\varphi_{i}(t)$.

(c) If no spikes reach the ith oscillator in time $\left(t_{1}, t_{2}\right)$ and the ith oscillator do not fire in time $\left(t_{1}, t_{2}\right)$, then $\varphi_{i}\left(t_{2}^{-}\right)=\varphi_{i}\left(t_{1}^{+}\right)+\left(t_{2}-t_{1}\right)$.

Remark 1 The assumption (A1) and Proposition 1(b) imply that $\varphi_{i}(t) \neq 0$ for all $t \geq 0$ and all $1 \leq i \leq N$. Thus, in our model, each phase variable $\varphi_{i}$ satisfies $\varphi_{i}(t) \in(0,1]$ for all $t \geq 0$.

\section{Definitions and notations}

In this paper, the concept of complete synchronization is defined as follows:

Definition 1 If there exists a $t_{0} \geq 0$ such that $\varphi_{i}(t)=\varphi_{j}(t)$ for all $t \geq t_{0}$, then we say that oscillator $i$ and $j$ can achieve complete synchronization or, for simplicity, synchronization; and say that oscillator $i$ and $j$ have already been completely synchronized at time $t_{0}$ or, for simplicity, synchronized.

Definition 2 If there exists a $t_{0} \geq 0$ such that $\varphi_{1}(t)=\varphi_{2}(t)=\cdots=\varphi_{N}(t)$ for all $t \geq t_{0}$, then we say that the pulse-coupled oscillator network can achieve complete synchronization or, for simplicity, synchronization; and say that the pulse-coupled oscillator network has already been completely synchronized at time $t_{0}$ or, for simplicity, synchronized.

Throughout the paper, the following notations will be used.

(i) $\quad F_{m}(\theta)=f^{-1}(\min [1, f(\theta)+m \varepsilon])$, where $m \in \mathbb{Z}^{+}$and $0 \leq \theta \leq 1$. Here, $\mathbb{Z}^{+}=\{z \in \mathbb{Z} \mid z \geq 0\}$.

(ii) $T_{i}=\left\{t \in \mathbb{R}^{+} \mid \mathrm{t}\right.$ is the time at which the $i$ th oscillator fires $\}, i=1, \ldots, N$. Here, $\mathbb{R}^{+}=\{z \in$ $\mathbb{R} \mid z \geq 0\}$.

(iii) $D_{i}(t)=\left\{\eta=t^{\prime}+\tau-t \mid t^{\prime} \in T_{i} \cap[t-\tau, t)\right\}, i=1, \ldots, N, t \geq 0$.

(iv) $D_{i}\left(t^{+}\right)=\left\{\eta=t^{\prime}+\tau-t \mid t^{\prime} \in T_{i} \cap(t-\tau, t]\right\}, i=1, \ldots, N, t \geq 0$, which is called the right-limit of $D_{i}(t)$ at time $t$. 
Remark 2 According to Proposition 1, if $m$ spikes reach the ith oscillator at time $t$, then $\varphi_{i}(t)=f^{-1}\left(\min \left[1, f\left(\varphi_{i}\left(t^{-}\right)\right)+m \varepsilon\right]\right)=F_{m}\left(\varphi_{i}\left(t^{-}\right)\right)$; if no spikes reach the $i$ th oscillator at time $t$, then $\varphi_{i}(t)=\varphi_{i}\left(t^{-}\right)=F_{0}\left(\varphi_{i}\left(t^{-}\right)\right)$. Therefore, for the convenience of later use, we introduced the notation $F_{m}(\theta)$.

Remark 3 The notation $T_{i}$ is called the firing-time set of the ith oscillator. Using the firingtime set $T_{i}$, we defined two other notations $D_{i}(t)$ and $D_{i}\left(t^{+}\right)$. They can be used to calculate the reaching time of the spikes which the ith oscillator has emitted, but the other oscillators have not received. From the definition of $D_{i}(t)$, one can see that for each $\eta \in D_{i}(t)$, there must be a spike of the ith oscillator, which will reach the other oscillators at time $t+\eta$. In particular, if $0 \in D_{i}(t)$, then there must be a spike of the ith oscillator, which reaches the other oscillators at time $t$.

Remark 4 The notations $D_{i}(t)$ and $D_{i}\left(t^{+}\right)$will play an important role in our discussion. For the pulse-coupled networks without transmission delays, instantaneous synchronization $\left(\varphi_{i}\left(t_{0}\right)=\right.$ $\left.\varphi_{j}\left(t_{0}\right)\right)$ shows that oscillator $i$ and $j$ have been completely synchronized at time $t_{0}\left(\varphi_{i}(t)=\varphi_{j}(t)\right.$ for all $\left.t \geq t_{0}\right)$, because the coupling is all-to-all and the oscillators have identical dynamics. But, for those with transmission delays, this criterion fails (instantaneous synchronization does not mean complete synchronization), because of the presence of delays. Fig.3 shows an example in which the criterion fails. One key role of $D_{i}(t)$ and $D_{i}\left(t^{+}\right)$in this paper is to judge whether the pulse-coupled oscillator network with delays has been completely synchronized at time $t_{0}$, when we have $\varphi_{1}\left(t_{0}\right)=\ldots=\varphi_{N}\left(t_{0}\right)$.

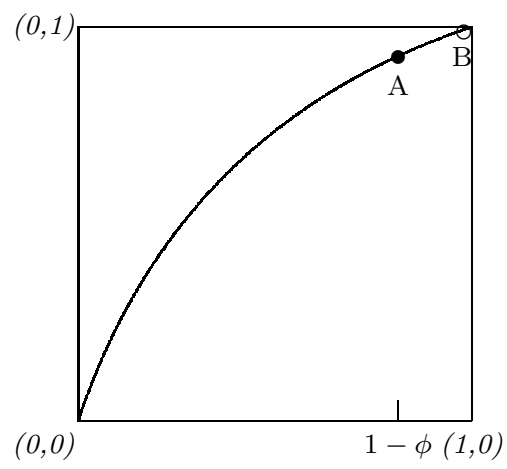

(a)

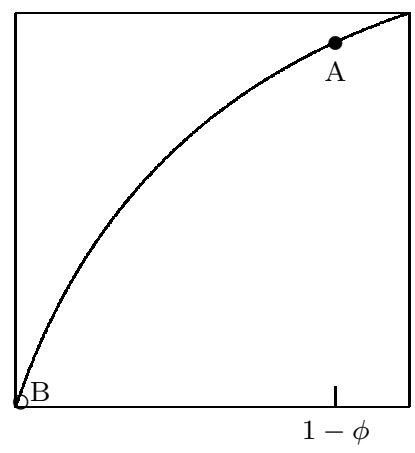

(b)

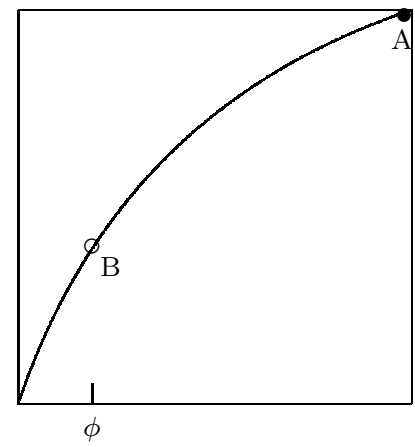

(c) 


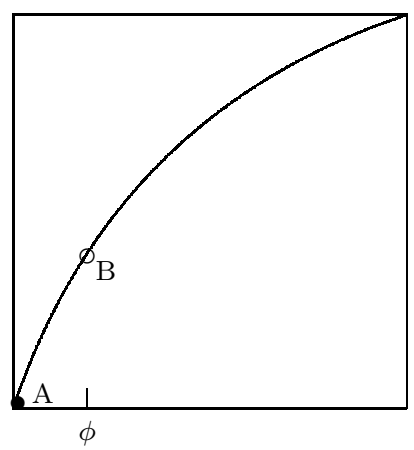

(d)

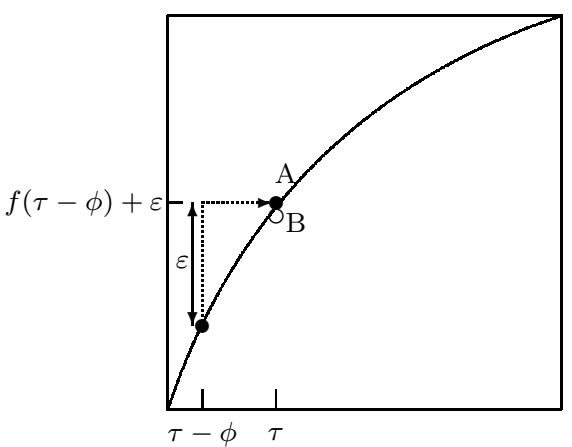

(e)

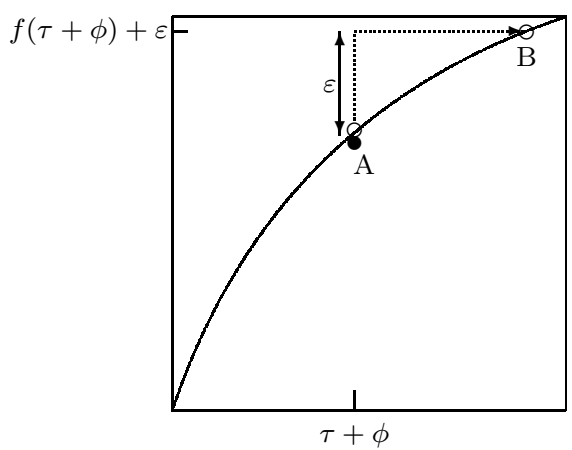

(f)

Fig.3: A system of two oscillators (A and B) governed by $x=f(\varphi)$, with $\tau$ and $\varepsilon$ satisfying $\varepsilon<f(\tau)<1$. The initial phases are chosen to be $\varphi_{A}(0)=1-\phi$ and $\varphi_{B}(0)=1$ such that $0<\phi<\tau$ and $f(\tau-\phi)+\varepsilon=f(\tau)$. (a) The state of the system at $t=0$. At the moment oscillator B emits a spike. (b) The state of the system just after $t=0$. (c) The state of the system at $t=\phi$. At the moment oscillator A emits a spike. (d) The state of the system just after $t=\phi$. (e) The state of the system at $t=\tau$. At the moment the spike emitted by $\mathrm{B}$ at $t=0$ reaches A. (f) The state of the system at $t=\tau+\phi$. At the moment the spike emitted by A at $t=\phi$ reaches B. Although equality $\varphi_{A}(s)=\varphi_{B}(s)$ holds for all $s \in[\tau, \tau+\phi)$, (f) shows that the system has not been synchronized at $t=\tau+\phi$.

Proposition $2 \quad F_{m}(\theta)$ has the following properties:

(a) $0 \leq F_{m}(\theta) \leq 1$ for all $0 \leq \theta \leq 1$ and all $m \in \mathbb{Z}^{+}$.

(b) If $\theta_{1} \leq \theta_{2}$, then $F_{m}\left(\theta_{1}\right) \leq F_{m}\left(\theta_{2}\right)$. In particular, if $\theta_{1}<\theta_{2}$ and $F_{m}\left(\theta_{1}\right)<1$, then $F_{m}\left(\theta_{1}\right)<F_{m}\left(\theta_{2}\right)$. (the monotonicity with respect to $\theta$ )

(c) If $m_{1} \leq m_{2}$, then $F_{m_{1}}(\theta) \leq F_{m_{2}}(\theta)$. In particular, if $m_{1}<m_{2}$ and $F_{m_{1}}(\theta)<1$, then $F_{m_{1}}(\theta)<F_{m_{2}}(\theta)$. (the monotonicity with respect to $m$ )

(d) When $0<F_{m}(\theta)<1$, the derivative of $F_{m}(\theta)$ with respect to $\theta$ exists, and $\frac{\mathrm{d} F_{m}(\theta)}{\mathrm{d} \theta}>1$.

(e) If $\theta, \delta \geq 0$ and $F_{m}(\theta+\delta)<1$, then $F_{m}(\theta)+\delta \leq F_{m}(\theta+\delta)$ and the equal sign holds if and only if $\delta=0$ or $m=0$, i.e., $m \delta=0$.

(f) $F_{n}\left(F_{m}(\theta)\right)=F_{m+n}(\theta)$ for all $0 \leq \theta \leq 1$ and all $m, n \in \mathbb{Z}^{+}$.

(g) If $\theta, \delta_{1}, \ldots, \delta_{n} \geq 0$ and $F_{m_{1}+\cdots+m_{n}}\left(\theta+\delta_{1}+\cdots+\delta_{n}\right)<1$, then $F_{m_{n}}\left(\cdots\left(F_{m_{2}}\left(F_{m_{1}}(\theta)+\delta_{1}\right)+\delta_{2}\right)+\cdots\right)+\delta_{n} \leq F_{m_{1}+\cdots+m_{n}}\left(\theta+\delta_{1}+\cdots+\delta_{n}\right)$ and the equal sign holds if and only if $m_{1} \delta_{1}=\cdots=m_{n} \delta_{n}=0$.

Remark 5 Property (d) comes from the concavity assumption of function $f$. Property (e) follows from (d), (f) is clear, and (g) follows from (e) and (f). 
Proposition $3 \quad D_{i}(t)$ and $D_{i}\left(t^{+}\right)$have the following properties:

(a) For each $\eta \in D_{i}(t)$, we have $0 \leq \eta<\tau$.

(a') For each $\eta \in D_{i}\left(t^{+}\right)$, we have $0<\eta \leq \tau$.

(b) For any $\eta_{1}, \eta_{2} \in D_{i}(t)$, we have $\left|\eta_{1}-\eta_{2}\right|<\tau$.

(b') For any $\eta_{1}, \eta_{2} \in D_{i}\left(t^{+}\right)$, we have $\left|\eta_{1}-\eta_{2}\right|<\tau$.

(c) If $\eta \in D_{i}(t)$, then $t-[\tau-\eta] \in T_{i}$, i.e., $\varphi_{i}(t-[\tau-\eta])=1$.

(c') If $\eta \in D_{i}\left(t^{+}\right)$, then $t-[\tau-\eta] \in T_{i}$, i.e., $\varphi_{i}(t-[\tau-\eta])=1$.

(d) If $\eta \in D_{i}(t)$ and $0<\eta<\tau$, then $\eta \in D_{i}\left(t^{+}\right)$too.

(d') If $\eta \in D_{i}\left(t^{+}\right)$and $0<\eta<\tau$, then $\eta \in D_{i}(t)$ too.

(e) If $\eta \in D_{i}(t)$, then $\eta-\omega \in D_{i}(t+\omega)$, where $\omega \in(\eta-\tau, \eta]$.

(e') If $\eta \in D_{i}\left(t^{+}\right)$, then $\eta-\omega \in D_{i}\left((t+\omega)^{+}\right)$, where $\omega \in[\eta-\tau, \eta)$.

\section{Properties of the pulse-coupled oscillator network with delayed excitatory coupling under the assumptions (A1) and (A2)}

It is difficult to prove the main theorem directly. The following lemmas are needed. Lemma 1 shows that under the assumptions (A1) and (A2), the time between any two firing activities of any oscillator is longer than $2 \tau$. This result is reasonable to fireflies, because the transmission delays of fireflies are relatively short and fireflies can not fire twice in such a short period of time. By Lemma 1, we obtain Lemma 2, which shows that under the assumptions (A1) and (A2), both $D_{i}(t)$ and $D_{i}\left(t^{+}\right)$have at most one element. In Lemmas 3-5, three criteria of synchronization of two oscillators are given. In Lemma 6 and Lemma 7, two special cases are discussed, respectively. The two lemmas show that the network can not achieve complete synchronization even if it has been divided into two cliques.

Lemma 1 If an oscillator fires at time $t_{1}$ and $t_{2}$ with $t_{1} \neq t_{2}$, then $\left|t_{1}-t_{2}\right|>2 \tau$.

Proof: Assume $t_{2}>t_{1}$ and that the oscillator is the $i$ th oscillator. Then we have $\varphi_{i}\left(t_{1}^{+}\right)=0$ and $\varphi_{i}\left(t_{2}\right)=1$. For $t_{1}, t_{2}$, there must be $t_{3}, t_{4} \in\left[t_{1}, t_{2}\right]$ such that the $i$ th oscillator fires at $t_{3}, t_{4}$ and does not fire in $\left(t_{3}, t_{4}\right)$. If we can prove $\left|t_{3}-t_{4}\right|>2 \tau$, then $\left|t_{1}-t_{2}\right|>2 \tau$ must hold. Therefore, without loss of generality, we can assume that the $i$ th oscillator does not fire in time $\left(t_{1}, t_{2}\right)$. 
Suppose $t_{2}-t_{1} \leq 2 \tau$.

Case 1: $t_{2}<\tau$.

Because of the presence of the transmission delay and the assumption (A1), no spikes can reach the $i$ th oscillator in time $\left(t_{1}, t_{2}\right] \subseteq(0, \tau)$. Then, according to Proposition $1, \varphi_{i}\left(t_{2}\right)=$ $\varphi_{i}\left(t_{2}^{-}\right)=\varphi_{i}\left(t_{1}^{+}\right)+\left(t_{2}-t_{1}\right)=t_{2}-t_{1}<\tau<1$, which contradicts that $\varphi_{i}\left(t_{2}\right)=1$. So, this case is impossible.

\section{Case 2: $t_{2}=\tau$.}

Because of the presence of the transmission delay and the assumption (A1), only the spikes that the other oscillators emit at time 0 can reach the $i$ th oscillator in time $\left(t_{1}, t_{2}\right]$. Assume that among the other oscillators, there are $m$ oscillators firing at time 0 . (Here, $m$ can be equal to zero. It means that among the other oscillators there are no oscillators firing at time 0 . For the case of $m=0$, the following proof holds true.) It is obvious that $0 \leq m \leq N-1$. According to Proposition 1 and the assumption (A2), we have

$$
\varphi_{i}\left(t_{2}^{-}\right)=\varphi_{i}\left(t_{1}^{+}\right)+\left(t_{2}-t_{1}\right)=\tau-t_{1} \leq \tau
$$

and

$$
\begin{aligned}
\varphi_{i}\left(t_{2}\right) & =f^{-1}\left(\min \left[1, f\left(\varphi_{i}\left(t_{2}^{-}\right)\right)+m \varepsilon\right]\right) \\
& =f^{-1}\left(\min \left[1, f\left(t_{2}-t_{1}\right)+m \varepsilon\right]\right) \\
& \leq f^{-1}(\min [1, f(\tau)+(N-1) \varepsilon]) \\
& \leq f^{-1}(\min [1, f(2 \tau)+N \varepsilon]) \\
& =f^{-1}(f(2 \tau)+N \varepsilon) \\
& <f^{-1}(1)=1
\end{aligned}
$$

which contradicts that $\varphi_{i}\left(t_{2}\right)=1$. So, this case is impossible.

Case 3: $t_{2}>\tau$.

Assume that there are $m=m_{1}+m_{2}+\cdots+m_{l}$ spikes which reach the $i$ th oscillator in time $\left(t_{1}, t_{2}\right]:$

$m_{1}$ spikes reach at time $t_{1}+\delta_{1}$;

$m_{2}$ spikes reach at time $t_{1}+\delta_{1}+\delta_{2}$;

- . . .

$m_{l}$ spikes reach at time $t_{1}+\delta_{1}+\cdots+\delta_{l}$. 
where $\delta_{1}, \ldots, \delta_{l}>0$, and $\delta_{1}+\cdots+\delta_{l} \leq t_{2}-t_{1}$. (Here, $m$ can be equal to zero. It means that there are no spikes reaching the $i$ th oscillator in time $\left(t_{1}, t_{2}\right]$. When $m=0$, we can let $l=1$, $m_{l}=0$ and $\delta_{l}$ be any value in the range $\left(0, t_{2}-t_{1}\right]$. Hence the following proof holds true for the case of $m=0$.)

Because the $i$ th oscillator does not fire in time $\left(t_{1}, t_{2}\right)$, according to Proposition 1 we have

$$
\begin{aligned}
& \varphi_{i}\left(\left[t_{1}+\delta_{1}\right]^{-}\right)=\varphi_{i}\left(t_{1}^{+}\right)+\delta_{1}=\delta_{1} \\
& \varphi_{i}\left(t_{1}+\delta_{1}\right)=F_{m_{1}}\left(\varphi_{i}\left(\left[t_{1}+\delta_{1}\right]^{-}\right)\right)=F_{m_{1}}\left(\delta_{1}\right) \\
& \varphi_{i}\left(\left[t_{1}+\delta_{1}+\delta_{2}\right]^{-}\right)=\varphi_{i}\left(t_{1}+\delta_{1}\right)+\delta_{2}=F_{m_{1}}\left(\delta_{1}\right)+\delta_{2} \\
& \varphi_{i}\left(t_{1}+\delta_{1}+\delta_{2}\right)=F_{m_{2}}\left(\varphi_{i}\left(\left[t_{1}+\delta_{1}+\delta_{2}\right]^{-}\right)\right)=F_{m_{2}}\left(F_{m_{1}}\left(\delta_{1}\right)+\delta_{2}\right) \\
& \cdots \cdots \\
& \varphi_{i}\left(\left[t_{1}+\delta_{1}+\cdots+\delta_{l}\right]^{-}\right)=\varphi_{i}\left(t_{1}+\delta_{1}+\cdots+\delta_{l-1}\right)+\delta_{l}=F_{m_{l-1}}\left(\cdots F_{m_{2}}\left(F_{m_{1}}\left(\delta_{1}\right)+\delta_{2}\right) \cdots+\delta_{l-1}\right)+\delta_{l} \\
& \varphi_{i}\left(t_{1}+\delta_{1}+\cdots+\delta_{l}\right)=F_{m_{l}}\left(\varphi_{i}\left(\left[t_{1}+\delta_{1}+\cdots+\delta_{l}\right]^{-}\right)\right)=F_{m_{l}}\left(F_{m_{l-1}}\left(\cdots F_{m_{2}}\left(F_{m_{1}}\left(\delta_{1}\right)+\delta_{2}\right) \cdots+\delta_{l-1}\right)+\delta_{l}\right)
\end{aligned}
$$

Because of the presence of the transmission delay and the assumption (A1), the spikes which reach the $i$ th oscillator in time $\left(t_{1}, t_{2}\right]$ are the ones that the other oscillators emit in time $\left(t_{1}-\right.$ $\left.\tau, t_{2}-\tau\right] \cap[0,+\infty)$. If all the other oscillators fire not more than once in $\left(t_{1}-\tau, t_{2}-\tau\right] \cap[0,+\infty)$, i.e., $m \leq N-1$, then it follows from the assumption (A2) and Proposition 2(g) that

$$
\begin{aligned}
\varphi_{i}\left(t_{2}\right) & =\varphi_{i}\left(t_{1}+\delta_{1}+\cdots+\delta_{l}\right)+\left[t_{2}-\left(t_{1}+\delta_{1}+\cdots+\delta_{l}\right)\right] \\
& =F_{m_{l}}\left(F_{m_{l-1}}\left(\cdots F_{m_{2}}\left(F_{m_{1}}\left(\delta_{1}\right)+\delta_{2}\right) \cdots+\delta_{l-1}\right)+\delta_{l}\right)+\left[t_{2}-\left(t_{1}+\delta_{1}+\cdots+\delta_{l}\right)\right] \\
& \leq F_{m_{1}+\cdots+m_{l}}\left(\delta_{1}+\cdots+\delta_{l}+\left[t_{2}-\left(t_{1}+\delta_{1}+\cdots+\delta_{l}\right)\right]\right) \\
& =F_{m_{1}+\cdots+m_{l}}\left(t_{2}-t_{1}\right) \\
& \leq F_{N}(2 \tau)<1
\end{aligned}
$$

which contradicts that $\varphi_{i}\left(t_{2}\right)=1$. Hence there must be some oscillator firing more than once in $\left(t_{1}-\tau, t_{2}-\tau\right] \cap[0,+\infty)$. It means that there must be time $t_{3}, t_{4}$ with $t_{3}<t_{4}$ and $t_{3}, t_{4} \in\left(t_{1}-\tau, t_{2}-\tau\right] \cap[0,+\infty)$ such that some oscillator fires at $t_{3}, t_{4}$ and does not fire in $\left(t_{3}, t_{4}\right)$. If $t_{4}>\tau$, as above, there must be time $t_{5}, t_{6}$ with $t_{5}<t_{6}$ and $t_{5}, t_{6} \in\left(t_{3}-\tau, t_{4}-\tau\right] \cap[0,+\infty)$ such that some oscillator fires at $t_{5}, t_{6}$ and does not fire in $\left(t_{5}, t_{6}\right)$. If $t_{6}>\tau, \cdots$ This leads to a finite sequence:

$$
\left(t_{1}, t_{2}\right) \rightarrow\left(t_{3}, t_{4}\right) \rightarrow \cdots \rightarrow\left(t_{2 k-1}, t_{2 k}\right) \rightarrow \cdots \rightarrow\left(t_{2 n-1}, t_{2 n}\right)
$$

which satisfies that

$$
\begin{aligned}
& t_{2(k-1)-1}-\tau<t_{2 k-1}<t_{2 k} \leq t_{2(k-1)}-\tau, k=2, \ldots, n, \\
& t_{2 k-1} \geq 0, k=1, \ldots, n \\
& t_{2 k}>\tau, k=1, \ldots, n-1, \text { and } t_{2 n} \leq \tau
\end{aligned}
$$


The last term of the sequence means that some oscillator fires at $t_{2 n-1}$ and $t_{2 n}$ and does not fire in $\left(t_{2 n-1}, t_{2 n}\right)$, with $0 \leq t_{2 n-1}<t_{2 n} \leq \tau$. By case 1 and case 2 , this is impossible. So, case 3 is impossible, too.

Therefore, we have $t_{2}-t_{1}>2 \tau$, which completes the proof.

By Lemma 1, we get Lemma 2.

Lemma 2 Under the assumptions (A1) and (A2), $D_{i}(t)$ and $D_{i}\left(t^{+}\right)$have the following properties:

(a) $D_{i}(t)=\{\eta\}$ with $0 \leq \eta<\tau$ or $D_{i}(t)=\emptyset$, for all $1 \leq i \leq N$ and all $t \geq 0$; i.e., set $D_{i}(t)$ has at most one element for all $1 \leq i \leq N$ and all $t \geq 0$.

(b) $D_{i}\left(t^{+}\right)=\{\eta\}$ with $0<\eta \leq \tau$ or $D_{i}\left(t^{+}\right)=\emptyset$, for all $1 \leq i \leq N$ and all $t \geq 0$; i.e., set $D_{i}\left(t^{+}\right)$ has at most one element for all $1 \leq i \leq N$ and all $t \geq 0$.

(c) If $D_{i}(t) \neq \emptyset$, then $0<\varphi_{i}(t)<1$.

(d) If $D_{i}(t)=\left\{\eta_{1}\right\}, D_{j}(t)=\left\{\eta_{2}\right\}$ and $\eta_{1}<\eta_{2}$, then $\varphi_{i}\left(t^{-}\right)>\varphi_{j}\left(t^{-}\right)$.

Proof: (a) Assume that there are $\eta_{1}, \eta_{2} \in D_{i}(t)$ with $\eta_{1} \neq \eta_{2}$. Then by Proposition 3(c) we have $\varphi_{i}\left(t-\left[\tau-\eta_{1}\right]\right)=\varphi_{i}\left(t-\left[\tau-\eta_{2}\right]\right)=1$, i.e., the $i$ th oscillator fires at time $t-\left[\tau-\eta_{1}\right]$ and $t-\left[\tau-\eta_{2}\right]$. However, it follows from Proposition 3(b) that $\left|\left[t-\left(\tau-\eta_{1}\right)\right]-\left[t-\left(\tau-\eta_{2}\right)\right]\right|=\left|\eta_{1}-\eta_{2}\right|<\tau$. This contradicts Lemma 1 . So, for any $\eta_{1}, \eta_{2} \in D_{i}(t)$, the equality $\eta_{1}=\eta_{2}$ must hold. It shows that $D_{i}(t)=\{\eta\}$ with $0 \leq \eta<\tau$, or $D_{i}(t)=\emptyset$.

(b) The proof is similar to that of (a).

(c) If $D_{i}(t) \neq \emptyset$, there must be a $\eta \in D_{i}(t)$ with $0 \leq \eta<\tau$. By Proposition 3(c), we have $\varphi_{i}(t-[\tau-\eta])=1$. Since $t-[t-(\tau-\eta)]=\tau-\eta<2 \tau$, by Lemma 1 , the $i$ th oscillator never fires at time $t$. It means $\varphi_{i}(t)<1$. Thus, from Remark 1 , we have $0<\varphi_{i}(t)<1$.

(d) Assume that there are $m=m_{1}+m_{2}+\cdots+m_{l}$ spikes which reach the $i$ th oscillator in time $\left(t-\left(\tau-\eta_{2}\right), t\right)$ :

$$
\begin{aligned}
& m_{1} \text { spikes reach at time } t-\left(\tau-\eta_{2}\right)+\delta_{1} ; \\
& m_{2} \text { spikes reach at time } t-\left(\tau-\eta_{2}\right)+\delta_{1}+\delta_{2}
\end{aligned}
$$

$m_{l}$ spikes reach at time $t-\left(\tau-\eta_{2}\right)+\delta_{1}+\cdots+\delta_{l}$.

where $\delta_{1}, \ldots, \delta_{l}>0$, and $\delta_{1}+\cdots+\delta_{l}<\tau-\eta_{2}$. (Here, $m$ can be equal to zero. It means that there are no spikes reaching the $i$ th oscillator in time $\left(t-\left(\tau-\eta_{2}\right), t\right)$. When $m=0$, we can let 
$l=1, m_{l}=0$ and $\delta_{l}$ be any value in the range $\left(0, \tau-\eta_{2}\right)$. Hence the following proof holds true for the case of $m=0$.)

Since $D_{i}(t)=\left\{\eta_{1}\right\}, D_{j}(t)=\left\{\eta_{2}\right\}$ and $\eta_{1}<\eta_{2}$, by Proposition 3(c) and 3(e) we have that $\varphi_{j}\left(t-\left(\tau-\eta_{2}\right)\right)=1, D_{i}(s)=\left\{\eta_{1}+(t-s)\right\}$ for all $s \in\left[t-\left(\tau-\eta_{2}\right), t\right]$ and $D_{j}(s)=\left\{\eta_{2}+(t-s)\right\}$ for all $s \in\left(t-\left(\tau-\eta_{2}\right), t\right]$.

From $D_{i}(s)=\left\{\eta_{1}+(t-s)\right\} \neq\{0\}$ and $D_{j}(s)=\left\{\eta_{2}+(t-s)\right\} \neq\{0\}$ for all $s \in\left(t-\left(\tau-\eta_{2}\right), t\right)$, it follows that oscillator $i$ and $j$ can not receive the spikes of each other in time $\left(t-\left(\tau-\eta_{2}\right), t\right)$, which means that the spikes reaching the $j$ th oscillator in time $\left(t-\left(\tau-\eta_{2}\right), t\right)$ are the $m$ spikes above.

Because $D_{i}(s)=\left\{\eta_{1}+(t-s)\right\} \neq \emptyset$ and $D_{j}(s)=\left\{\eta_{2}+(t-s)\right\} \neq \emptyset$ for all $s \in\left(t-\left(\tau-\eta_{2}\right), t\right)$, from Lemma 2(c), it follows that $0<\varphi_{i}(s)<1$ and $0<\varphi_{j}(s)<1$ for all $s \in\left(t-\left(\tau-\eta_{2}\right), t\right)$, that is, both oscillator $i$ and $j$ do not fire in time $\left(t-\left(\tau-\eta_{2}\right), t\right)$.

Since $D_{i}\left(t-\left(\tau-\eta_{2}\right)\right)=\left\{\tau+\eta_{1}-\eta_{2}\right\} \neq \emptyset$, by Lemma $2(\mathrm{c})$ we have $0<\varphi_{i}\left(t-\left(\tau-\eta_{2}\right)\right)<1$. Denote $\phi=\varphi_{i}\left(t-\left(\tau-\eta_{2}\right)\right)$.

Therefore, according to Proposition 1, we have

$$
\begin{aligned}
& \varphi_{i}\left(\left[t-\left(\tau-\eta_{2}\right)+\delta_{1}\right]^{-}\right)=\phi+\delta_{1} \\
& \varphi_{i}\left(t-\left(\tau-\eta_{2}\right)+\delta_{1}\right)=F_{m_{1}}\left(\phi+\delta_{1}\right) \\
& \varphi_{i}\left(\left[t-\left(\tau-\eta_{2}\right)+\delta_{1}+\delta_{2}\right]^{-}\right)=F_{m_{1}}\left(\phi+\delta_{1}\right)+\delta_{2} \\
& \varphi_{i}\left(t-\left(\tau-\eta_{2}\right)+\delta_{1}+\delta_{2}\right)=F_{m_{2}}\left(F_{m_{1}}\left(\phi+\delta_{1}\right)+\delta_{2}\right) \\
& \cdots \cdots \\
& \varphi_{i}\left(\left[t-\left(\tau-\eta_{2}\right)+\delta_{1}+\cdots+\delta_{l}\right]^{-}\right)=F_{m_{l-1}}\left(\cdots F_{m_{2}}\left(F_{m_{1}}\left(\phi+\delta_{1}\right)+\delta_{2}\right) \cdots+\delta_{l-1}\right)+\delta_{l} \\
& \varphi_{i}\left(t-\left(\tau-\eta_{2}\right)+\delta_{1}+\cdots+\delta_{l}\right)=F_{m_{l}}\left(F_{m_{l-1}}\left(\cdots F_{m_{2}}\left(F_{m_{1}}\left(\phi+\delta_{1}\right)+\delta_{2}\right) \cdots+\delta_{l-1}\right)+\delta_{l}\right)
\end{aligned}
$$

and

$$
\begin{aligned}
& \varphi_{j}\left(\left[t-\left(\tau-\eta_{2}\right)+\delta_{1}\right]^{-}\right)=\delta_{1} \\
& \varphi_{j}\left(t-\left(\tau-\eta_{2}\right)+\delta_{1}\right)=F_{m_{1}}\left(\delta_{1}\right) \\
& \varphi_{j}\left(\left[t-\left(\tau-\eta_{2}\right)+\delta_{1}+\delta_{2}\right]^{-}\right)=F_{m_{1}}\left(\delta_{1}\right)+\delta_{2} \\
& \varphi_{j}\left(t-\left(\tau-\eta_{2}\right)+\delta_{1}+\delta_{2}\right)=F_{m_{2}}\left(F_{m_{1}}\left(\delta_{1}\right)+\delta_{2}\right) \\
& \cdots \cdots \\
& \varphi_{j}\left(\left[t-\left(\tau-\eta_{2}\right)+\delta_{1}+\cdots+\delta_{l}\right]^{-}\right)=F_{m_{l-1}}\left(\cdots F_{m_{2}}\left(F_{m_{1}}\left(\delta_{1}\right)+\delta_{2}\right) \cdots+\delta_{l-1}\right)+\delta_{l} \\
& \varphi_{j}\left(t-\left(\tau-\eta_{2}\right)+\delta_{1}+\cdots+\delta_{l}\right)=F_{m_{l}}\left(F_{m_{l-1}}\left(\cdots F_{m_{2}}\left(F_{m_{1}}\left(\delta_{1}\right)+\delta_{2}\right) \cdots+\delta_{l-1}\right)+\delta_{l}\right)
\end{aligned}
$$


By Proposition 2(b), we have

$$
\begin{aligned}
& \varphi_{i}\left(t-\left(\tau-\eta_{2}\right)+\delta_{1}\right)=F_{m_{1}}\left(\phi+\delta_{1}\right)>F_{m_{1}}\left(\delta_{1}\right)=\varphi_{j}\left(t-\left(\tau-\eta_{2}\right)+\delta_{1}\right) \\
& \varphi_{i}\left(t-\left(\tau-\eta_{2}\right)+\delta_{1}+\delta_{2}\right)=F_{m_{2}}\left(F_{m_{1}}\left(\phi+\delta_{1}\right)+\delta_{2}\right)>F_{m_{2}}\left(F_{m_{1}}\left(\delta_{1}\right)+\delta_{2}\right)=\varphi_{j}\left(t-\left(\tau-\eta_{2}\right)+\delta_{1}+\delta_{2}\right) \\
& \ldots \ldots \\
& \begin{aligned}
\varphi_{i}\left(t-\left(\tau-\eta_{2}\right)+\delta_{1}+\cdots+\delta_{l}\right) & =F_{m_{l}}\left(\cdots F_{m_{2}}\left(F_{m_{1}}\left(\phi+\delta_{1}\right)+\delta_{2}\right) \cdots+\delta_{l}\right) \\
& >F_{m_{l}}\left(\cdots F_{m_{2}}\left(F_{m_{1}}\left(\delta_{1}\right)+\delta_{2}\right) \cdots+\delta_{l}\right)=\varphi_{j}\left(t-\left(\tau-\eta_{2}\right)+\delta_{1}+\cdots+\delta_{l}\right)
\end{aligned}
\end{aligned}
$$

Therefore,

$$
\begin{aligned}
\varphi_{i}\left(t^{-}\right) & =\varphi_{i}\left(t-\left(\tau-\eta_{2}\right)+\delta_{1}+\cdots+\delta_{l}\right)+\left\{t-\left[t-\left(\tau-\eta_{2}\right)+\delta_{1}+\cdots+\delta_{l}\right]\right\} \\
& >\varphi_{j}\left(t-\left(\tau-\eta_{2}\right)+\delta_{1}+\cdots+\delta_{l}\right)+\left\{t-\left[t-\left(\tau-\eta_{2}\right)+\delta_{1}+\cdots+\delta_{l}\right]\right\} \\
& =\varphi_{j}\left(t^{-}\right)
\end{aligned}
$$

In the following (Lemmas 3-5), three synchronization criteria of two oscillators will be given.

Lemma 3 Oscillator $i$ and $j$ have been synchronized at time $t_{0}$, if and only if the two oscillators satisfy $\varphi_{i}\left(t_{0}\right)=\varphi_{j}\left(t_{0}\right)$ and $D_{i}\left(t_{0}^{+}\right)=D_{j}\left(t_{0}^{+}\right)$.

Proof: (1) Suppose that $\varphi_{i}\left(t_{0}\right)=\varphi_{j}\left(t_{0}\right)$ and $D_{i}\left(t_{0}^{+}\right)=D_{j}\left(t_{0}^{+}\right)$.

It means that the two oscillators have acted as one, because their dynamics are identical and they are coupled in the same way to all the other oscillators. That is, oscillator $i$ and $j$ have been synchronized at $t_{0}$.

(2) Suppose that oscillator $i$ and $j$ have been synchronized at $t_{0}$, namely, $\varphi_{i}(t)=\varphi_{j}(t)$ for all $t \geq t_{0}$.

We only need to prove $D_{i}\left(t_{0}^{+}\right)=D_{j}\left(t_{0}^{+}\right)$. Assume $D_{i}\left(t_{0}^{+}\right) \neq D_{j}\left(t_{0}^{+}\right)$.

It is clear that $D_{i}\left(t_{0}^{+}\right) \neq \emptyset$ or $D_{j}\left(t_{0}^{+}\right) \neq \emptyset$. Without loss of generality, we let $D_{i}\left(t_{0}^{+}\right) \neq \emptyset$. By Lemma 2(b), we have $D_{i}\left(t_{0}^{+}\right)=\{\eta\} \neq D_{j}\left(t_{0}^{+}\right)$with $0<\eta \leq \tau$. Then by Proposition 3 , we get $D_{i}\left(t_{0}+\eta\right)=\{0\} \neq D_{j}\left(t_{0}+\eta\right)$, which means that a spike of oscillator $i$ will reach oscillator $j$ at time $t_{0}+\eta$, but no spikes of oscillator $j$ will reach oscillator $i$ at time $t_{0}+\eta$. We let $m_{i}$ be the number of the spikes which reach oscillator $i$ at time $t_{0}+\eta$, and $m_{j}$ be the number of the spikes which reach oscillator $j$ at time $t_{0}+\eta$. So, the equality $m_{i}=m_{j}-1$ holds, because the two oscillators are coupled in the same way to all the other oscillators. It is obvious that $\varphi_{i}\left(\left[t_{0}+\eta\right]^{-}\right)=$ $\varphi_{j}\left(\left[t_{0}+\eta\right]^{-}\right)$, because $\varphi_{i}(t)=\varphi_{j}(t)$ for all $t \geq t_{0}$. Then, according to Proposition 1 , we have 
$\varphi_{i}\left(t_{0}+\eta\right)=f^{-1}\left(\min \left[1, f\left(\varphi_{i}\left(\left[t_{0}+\eta\right]^{-}\right)\right)+m_{i} \varepsilon\right]\right) \neq f^{-1}\left(\min \left[1, f\left(\varphi_{j}\left(\left[t_{0}+\eta\right]^{-}\right)\right)+m_{j} \varepsilon\right]\right)=\varphi_{j}\left(t_{0}+\eta\right)$, unless $f\left(\varphi_{i}\left(\left[t_{0}+\eta\right]^{-}\right)\right)+m_{i} \varepsilon \geq 1$ and $f\left(\varphi_{j}\left(\left[t_{0}+\eta\right]^{-}\right)\right)+m_{j} \varepsilon \geq 1$, i.e., $\varphi_{i}\left(t_{0}+\eta\right)=\varphi_{j}\left(t_{0}+\eta\right)=1$. However, by $D_{i}\left(t_{0}+\eta\right)=\{0\} \neq \emptyset$ and Lemma $2(\mathrm{c})$, we have $\varphi_{i}\left(t_{0}+\eta\right)<1$. This is a contradiction. Thus, $D_{i}\left(t_{0}^{+}\right)=D_{j}\left(t_{0}^{+}\right)$.

Lemma 4 If $D_{i}\left(t_{0}\right)=D_{j}\left(t_{0}\right)=\{\eta\}$ with $0 \leq \eta<\tau$, then oscillator $i$ and $j$ have been synchronized at time $t_{0}-(\tau-\eta)$; conversely, if there exists a $t_{1}<t_{0}$ such that oscillator $i$ and $j$ have been synchronized at time $t_{1}$, then $D_{i}\left(t_{0}\right)=D_{j}\left(t_{0}\right)$.

Proof: (1) Suppose that $D_{i}\left(t_{0}\right)=D_{j}\left(t_{0}\right)=\{\eta\}$ with $0 \leq \eta<\tau$.

From Proposition 3, we have $D_{i}\left(\left[t_{0}-(\tau-\eta)\right]^{+}\right)=D_{j}\left(\left[t_{0}-(\tau-\eta)\right]^{+}\right)=\{\tau\}$ and $\varphi_{i}\left(t_{0}-(\tau-\eta)\right)=$ $\varphi_{j}\left(t_{0}-(\tau-\eta)\right)=1$. By Lemma 3, oscillator $i$ and $j$ have been synchronized at time $t_{0}-(\tau-\eta)$.

(2) Suppose that there exists a $t_{1}<t_{0}$ such that oscillator $i$ and $j$ have been synchronized at time $t_{1}$.

Assume $D_{i}\left(t_{0}\right) \neq D_{j}\left(t_{0}\right)$. It is clear that $D_{i}\left(t_{0}\right) \neq \emptyset$ or $D_{j}\left(t_{0}\right) \neq \emptyset$. Without loss of generality, we let $D_{i}\left(t_{0}\right) \neq \emptyset$. By Lemma 2(a), we have $D_{i}\left(t_{0}\right)=\{\eta\} \neq D_{j}\left(t_{0}\right)$ with $0 \leq \eta<\tau$. If follows from Proposition 3 that $D_{i}\left(t_{0}+\eta\right)=\{0\} \neq D_{j}\left(t_{0}+\eta\right)$. Since oscillator $i$ and $j$ have been synchronized at time $t_{1}$ with $t_{1}<t_{0}$, by an argument similar to that used in the second part of the proof of Lemma 3 , we can obtain $\varphi_{i}\left(t_{0}+\eta\right)=\varphi_{j}\left(t_{0}+\eta\right)=1$. However, by $D_{i}\left(t_{0}+\eta\right)=\{0\} \neq \emptyset$ and Lemma 2(c), we have $\varphi_{i}\left(t_{0}+\eta\right)<1$. This is a contradiction. Thus, $D_{i}\left(t_{0}\right)=D_{j}\left(t_{0}\right)$.

Lemma 5 Oscillator $i$ and $j$ have been synchronized at time $t_{0}>0$ and they have not been synchronized at time $t$ for all $0 \leq t<t_{0}$, if and only if the two oscillators satisfy one of the following cases:

(a) $\varphi_{i}\left(t_{0}^{-}\right) \neq \varphi_{j}\left(t_{0}^{-}\right), D_{i}\left(t_{0}\right)=D_{j}\left(t_{0}\right)=\emptyset$ and $\varphi_{i}\left(t_{0}\right)=\varphi_{j}\left(t_{0}\right)=1$.

(b) $\varphi_{i}\left(t_{0}^{-}\right) \neq \varphi_{j}\left(t_{0}^{-}\right), D_{i}\left(t_{0}\right)=\{0\}, D_{j}\left(t_{0}\right)=\emptyset$ and $\varphi_{i}\left(t_{0}\right)=\varphi_{j}\left(t_{0}\right)<1$.

(c) $\varphi_{i}\left(t_{0}^{-}\right) \neq \varphi_{j}\left(t_{0}^{-}\right), D_{i}\left(t_{0}\right)=\emptyset, D_{j}\left(t_{0}\right)=\{0\}$ and $\varphi_{i}\left(t_{0}\right)=\varphi_{j}\left(t_{0}\right)<1$.

Proof: (1) Suppose that oscillator $i$ and $j$ have been synchronized at $t_{0}$ and that they have not been synchronized at $t$ for all $0 \leq t<t_{0}$.

We have $\varphi_{i}\left(t_{0}\right)=\varphi_{j}\left(t_{0}\right)$, because oscillator $i$ and $j$ have been synchronized at $t_{0}$. Assume that $\varphi_{i}\left(t_{0}^{-}\right)=\varphi_{j}\left(t_{0}^{-}\right)$. From Lemma 1 , there must be a $\delta>0$ such that no oscillators fire and no spikes reach in time $\left(t_{0}-\delta, t_{0}\right)$. It means that $\mathrm{d} \varphi_{i}(t) / \mathrm{d} t=\mathrm{d} \varphi_{j}(t) / \mathrm{d} t=1$ for all $t \in\left(t_{0}-\delta, t_{0}\right)$. Thus, we get $\varphi_{i}(t)=\varphi_{j}(t)$ for all $t \geq t_{0}-\delta / 2$. This contradicts that oscillator $i$ and $j$ have not been synchronized at $t$ for all $0 \leq t<t_{0}$. So, $\varphi_{i}\left(t_{0}^{-}\right) \neq \varphi_{j}\left(t_{0}^{-}\right)$. 
If no spikes reach both oscillator $i$ and $j$ at time $t_{0}$, then it follows from $\varphi_{i}\left(t_{0}^{-}\right) \neq \varphi_{j}\left(t_{0}^{-}\right)$that $\varphi_{i}\left(t_{0}\right)=\varphi_{i}\left(t_{0}^{-}\right) \neq \varphi_{j}\left(t_{0}^{-}\right)=\varphi_{j}\left(t_{0}\right)$. It contradicts that oscillator $i$ and $j$ have been synchronized at $t_{0}$. Therefore, there must be a spike which reaches at least one of the two oscillators at time $t_{0}$, i.e, there must be an oscillator $k$ such that $D_{k}\left(t_{0}\right)=\{0\}$. (This is an important property of pulsecoupled networks.) Let $K$ be a set of oscillators: oscillator $k^{\prime} \in K$ if and only if $D_{k^{\prime}}\left(t_{0}\right)=\{0\}$. And let $m$ be the number of the oscillators in $K$.

Case 1: oscillator $i, j \notin K$.

Then $f^{-1}\left(\min \left[1, f\left(\varphi_{i}\left(t_{0}^{-}\right)\right)+m \varepsilon\right]\right)=\varphi_{i}\left(t_{0}\right)=\varphi_{j}\left(t_{0}\right)=f^{-1}\left(\min \left[1, f\left(\varphi_{j}\left(t_{0}^{-}\right)\right)+m \varepsilon\right]\right)$. But $\varphi_{i}\left(t_{0}^{-}\right) \neq \varphi_{j}\left(t_{0}^{-}\right)$. So, $\varphi_{i}\left(t_{0}\right)=\varphi_{j}\left(t_{0}\right)=1$. By Lemma $2(\mathrm{c})$, we get $D_{i}\left(t_{0}\right)=D_{j}\left(t_{0}\right)=\emptyset$. Then case (a) holds.

Case 2: oscillator $i \in K$ and oscillator $j \notin K$.

Then we have $D_{i}\left(t_{0}\right)=\{0\} \neq D_{j}\left(t_{0}\right) . D_{i}\left(t_{0}\right)=\{0\}$ shows that $D_{i}\left(t_{0}^{+}\right)=\emptyset$ and $\varphi_{i}\left(t_{0}\right)<1$. Since oscillator $i$ and $j$ have been synchronized at $t_{0}$, it follows from Lemma 3 that $D_{j}\left(t_{0}^{+}\right)=$ $D_{i}\left(t_{0}^{+}\right)=\emptyset$, which means $D_{j}\left(t_{0}\right)=\emptyset$ or $\{0\}$. But $D_{j}\left(t_{0}\right) \neq\{0\}$. So, $D_{j}\left(t_{0}\right)=\emptyset$. Then case (b) holds.

Case 3: oscillator $i \notin K$ and oscillator $j \in K$.

It is similar to case 2 . And case (c) holds.

Case 4: oscillator $i, j \in K$.

Then $D_{i}\left(t_{0}\right)=D_{j}\left(t_{0}\right)=\{0\}$. By Lemma 4 , oscillator $i$ and $j$ have been synchronized at time $t_{0}-\tau<t_{0}$, which contradicts that oscillator $i$ and $j$ have not been synchronized at $t$ for all $0 \leq t<t_{0}$. Therefore, this case is impossible.

(2) Suppose that one of the three cases (a), (b) and (c) holds. Then we have $\varphi_{i}\left(t_{0}\right)=\varphi_{j}\left(t_{0}\right)$ and $D_{i}\left(t_{0}^{+}\right)=D_{j}\left(t_{0}^{+}\right)$. From Lemma 3, oscillator $i$ and $j$ have been synchronized at $t_{0}$. If there exists a $t^{\prime}<t_{0}$ such that oscillator $i$ and $j$ have been synchronized at $t^{\prime}$, then $\varphi_{i}\left(t_{0}^{-}\right)=\varphi_{j}\left(t_{0}^{-}\right)$. This is a contradiction. So, oscillator $i$ and $j$ have not been synchronized at $t$ for all $0 \leq t<t_{0}$.

Two special cases are discussed in the following two lemmas, which show that the network can not achieve synchronization even if it has been divided into two cliques.

Lemma 6 If there exists a $t_{0} \geq 0$ such that $\varphi_{1}\left(t_{0}\right)=\cdots=\varphi_{m}\left(t_{0}\right)=1-\varphi(0<\varphi<1)$, $D_{1}\left(t_{0}\right)=\cdots=D_{m}\left(t_{0}\right)=\emptyset$ and $\varphi_{m+1}\left(t_{0}\right)=\cdots=\varphi_{N}\left(t_{0}\right)=1, D_{m+1}\left(t_{0}\right)=\cdots=D_{N}\left(t_{0}\right)=\emptyset$, where $1 \leq m<N$, then the pulse-coupled oscillator network can not achieve synchronization. 
Proof: Let $n=N-m$.

$\varphi_{1}\left(t_{0}\right)=\cdots=\varphi_{m}\left(t_{0}\right)=1-\varphi(0<\varphi<1), D_{1}\left(t_{0}\right)=\cdots=D_{m}\left(t_{0}\right)=\emptyset$ and $\varphi_{m+1}\left(t_{0}\right)=\cdots=$ $\varphi_{N}\left(t_{0}\right)=1, D_{m+1}\left(t_{0}\right)=\cdots=D_{N}\left(t_{0}\right)=\emptyset$ show that oscillator $1, \ldots, m$ have been synchronized at $t_{0}$, and oscillator $m+1, \ldots, N$ have also been synchronized at $t_{0}$. Therefore, we only need to discuss two oscillators, the $i$ th oscillator and the $j$ th oscillator $(1 \leq i \leq m, m+1 \leq j \leq N)$. The synchronization of the whole network is equivalent to the synchronization of oscillator $i$ and $j$.

According to the model rules, we get the following tables of $\varphi_{i}(t), D_{i}(t), \varphi_{j}(t)$ and $D_{j}(t)$ :

Case 1: $\varphi<\tau$.

\begin{tabular}{|l|l|l|l|l|}
\hline time $t$ & $\varphi_{i}(t)$ & $D_{i}(t)$ & $\varphi_{j}(t)$ & $D_{j}(t)$ \\
\hline \hline$t_{0}$ & $1-\varphi$ & $\emptyset$ & 1 & $\emptyset$ \\
\hline$t_{0}^{+}$ & $1-\varphi$ & $\emptyset$ & 0 & $\{\tau\}$ \\
\hline$t_{0}+\varphi$ & 1 & $\emptyset$ & $\varphi$ & $\{\tau-\varphi\}$ \\
\hline$\left(t_{0}+\varphi\right)^{+}$ & 0 & $\{\tau\}$ & $\varphi$ & $\{\tau-\varphi\}$ \\
\hline$t_{0}+\tau$ & $F_{n}(\tau-\varphi)$ & $\{\varphi\}$ & $F_{n-1}(\tau)$ & $\{0\}$ \\
\hline$\left(t_{0}+\tau\right)^{+}$ & $F_{n}(\tau-\varphi)$ & $\{\varphi\}$ & $F_{n-1}(\tau)$ & $\emptyset$ \\
\hline$t_{0}+\tau+\varphi$ & $F_{m-1}\left(F_{n}(\tau-\varphi)+\varphi\right)$ & $\{0\}$ & $F_{m}\left(F_{n-1}(\tau)+\varphi\right)$ & $\emptyset$ \\
\hline$\left(t_{0}+\tau+\varphi\right)^{+}$ & $F_{m-1}\left(F_{n}(\tau-\varphi)+\varphi\right)$ & $\emptyset$ & $F_{m}\left(F_{n-1}(\tau)+\varphi\right)$ & $\emptyset$ \\
\hline$t_{0}+\tau+\varphi+$ & $1-\left[F_{m}\left(F_{n-1}(\tau)+\varphi\right)\right.$ & $\emptyset$ & 1 & $\emptyset$ \\
$1-F_{m}\left(F_{n-1}(\tau)+\varphi\right)$ & $\left.-F_{m-1}\left(F_{n}(\tau-\varphi)+\varphi\right)\right]$ & & & \\
\hline
\end{tabular}

Case 2: $\varphi \geq \tau$.

\begin{tabular}{|l|l|l|l|l|}
\hline time $t$ & $\varphi_{i}(t)$ & $D_{i}(t)$ & $\varphi_{j}(t)$ & $D_{j}(t)$ \\
\hline \hline$t_{0}$ & $1-\varphi$ & $\emptyset$ & 1 & $\emptyset$ \\
\hline$t_{0}^{+}$ & $1-\varphi$ & $\emptyset$ & 0 & $\{\tau\}$ \\
\hline$t_{0}+\tau$ & $F_{n}(1-\varphi+\tau)$ & $\emptyset$ & $F_{n-1}(\tau)$ & $\{0\}$ \\
\hline$\left(t_{0}+\tau\right)^{+}$ & $F_{n}(1-\varphi+\tau)$ & $\emptyset$ & $F_{n-1}(\tau)$ & $\emptyset$ \\
\hline$t_{0}+\tau+$ & 1 & $\emptyset$ & $1-\left[F_{n}(1-\varphi+\tau)\right.$ & $\emptyset$ \\
$1-F_{n}(1-\varphi+\tau)$ & & & $\left.-F_{n-1}(\tau)\right]$ & \\
\hline
\end{tabular}

Let

$$
\begin{array}{ll}
G_{1}(\theta)=F_{m}\left(F_{n-1}(\tau)+\theta\right)-F_{m-1}\left(F_{n}(\tau-\theta)+\theta\right) & 0 \leq \theta<\tau \\
G_{2}(\theta)=F_{n}\left(F_{m-1}(\tau)+\theta\right)-F_{n-1}\left(F_{m}(\tau-\theta)+\theta\right) & 0 \leq \theta<\tau \\
G_{3}(\theta)=F_{n}(1-\theta+\tau)-F_{n-1}(\tau) & \tau \leq \theta<1 \\
G_{4}(\theta)=F_{m}(1-\theta+\tau)-F_{m-1}(\tau) & \tau \leq \theta<1
\end{array}
$$


Define map of the form

$$
G(\theta, P, Q)= \begin{cases}\left(G_{1}(\theta), P, Q\right) & \text { if } \quad 0 \leq \theta<\tau, P=m, Q=n \\ \left(G_{2}(\theta), P, Q\right) & \text { if } \quad 0 \leq \theta<\tau, P=n, Q=m \\ \left(G_{3}(\theta), Q, P\right) & \text { if } \tau \leq \theta<1, P=m, Q=n \\ \left(G_{4}(\theta), Q, P\right) & \text { if } \tau \leq \theta<1, P=n, Q=m\end{cases}
$$

where $0 \leq \theta<1, P=m$ or $n, Q=m$ or $n$.

Then the difference between the phase variables $\varphi_{i}$ and $\varphi_{j}$ can be shown by the following sequence:

$$
(\varphi, m, n) \rightarrow G(\varphi, m, n) \rightarrow G^{2}(\varphi, m, n) \rightarrow \cdots
$$

If the pulse-coupled oscillator network can achieve synchronization, then there must be a $p \in \mathbb{N}$ such that $G^{p}(\varphi, m, n)=(0, m, n)$ or $(0, n, m)$. In the following, we will prove that such $p$ does not exist.

When $0<\theta<\tau$, we have $0<F_{n}(\tau-\theta)<1,0<F_{m}\left(F_{n-1}(\tau)+\theta\right)<1$ and $0<F_{m-1}\left(F_{n}(\tau-\right.$ $\theta)+\theta)<1$. From Proposition $2(\mathrm{~d})$, it follows that $F_{n}^{\prime}(\tau-\theta)>1, F_{m}^{\prime}\left(F_{n-1}(\tau)+\theta\right)>1$ and $F_{m-1}^{\prime}\left(F_{n}(\tau-\theta)+\theta\right)>1$, for all $0<\theta<\tau$. Therefore,

$$
\frac{d G_{1}(\theta)}{d \theta}=F_{m}^{\prime}\left(F_{n-1}(\tau)+\theta\right)-F_{m-1}^{\prime}\left(F_{n}(\tau-\theta)+\theta\right) \cdot\left[-F_{n}^{\prime}(\tau-\theta)+1\right]>1
$$

for all $0<\theta<\tau$. Then from $G_{1}(0)=0$, it follows that $G_{1}(\theta)>0$ for all $0<\theta<\tau$.

When $\tau \leq \theta<1$, since $1-\theta+\tau>\tau$ and $F_{n-1}(\tau)<1$, by Proposition 2(b) and 2(c) we have that

$$
G_{3}(\theta)=F_{n}(1-\theta+\tau)-F_{n-1}(\tau)>0
$$

for all $\tau \leq \theta<1$.

Similarly, we can get that $G_{2}(\theta)>0$ for all $0<\theta<\tau$ and $G_{4}(\theta)>0$ for all $\tau \leq \theta<1$.

Thus, there does not exist $p \in \mathbb{N}$ such that $G^{p}(\varphi, m, n)=(0, m, n)$ or $(0, n, m)$. It means that the pulse-coupled oscillator network can not achieve synchronization.

Lemma 7 If there exists a $t_{0} \geq 0$ such that $\varphi_{1}\left(t_{0}\right)=\cdots=\varphi_{m}\left(t_{0}\right)=\varphi \quad(0<\varphi<1)$, $D_{1}\left(t_{0}\right)=\cdots=D_{m}\left(t_{0}\right)=\{\eta\} \quad(0<\eta<\tau)$ and $\varphi_{m+1}\left(t_{0}\right)=\cdots=\varphi_{N}\left(t_{0}\right)=1, D_{m+1}\left(t_{0}\right)=\cdots=$ $D_{N}\left(t_{0}\right)=\emptyset$, then the pulse-coupled oscillator network can not achieve synchronization. 
Proof: Similarly as in the proof of Lemma 6, we only need to discuss two oscillator $i$ and $j$, with $1 \leq i \leq m$ and $m+1 \leq j \leq N$.

Let $n=N-m$.

By $D_{1}\left(t_{0}\right)=\cdots=D_{i-1}\left(t_{0}\right)=D_{i+1}\left(t_{0}\right)=\cdots=D_{m}\left(t_{0}\right)=\{\eta\}(0<\eta<\tau)$ and Lemma 1 , oscillator $1, \ldots, i-1, i+1, \ldots, m$ do not fire in time $\left[\max \left(0, t_{0}-(\tau-\eta)-\tau\right), \max \left(0, t_{0}-\right.\right.$ $\tau)]$. By $\varphi_{m+1}\left(t_{0}\right)=\cdots=\varphi_{N}\left(t_{0}\right)=1$ and Lemma 1 , oscillator $m+1, \ldots, N$ do not fire in $\left[\max \left(0, t_{0}-(\tau-\eta)-\tau\right), \max \left(0, t_{0}-\tau\right)\right]$, too. Therefore, no spikes can reach the $i$ th oscillator in time $\left[t_{0}-(\tau-\eta), t_{0}\right]$. Then we have $\varphi_{i}\left(t_{0}\right)=\tau-\eta$, i.e., $\eta=\tau-\varphi$.

Now we have the following table of $\varphi_{i}(t), D_{i}(t), \varphi_{j}(t)$ and $D_{j}(t)$ :

\begin{tabular}{|l|l|l|l|l|}
\hline time $t$ & $\varphi_{i}(t)$ & $D_{i}(t)$ & $\varphi_{j}(t)$ & $D_{j}(t)$ \\
\hline \hline$t_{0}$ & $\varphi$ & $\{\tau-\varphi\}$ & 1 & $\emptyset$ \\
\hline$t_{0}^{+}$ & $\varphi$ & $\{\tau-\varphi\}$ & 0 & $\{\tau\}$ \\
\hline$t_{0}+\tau-\varphi$ & $F_{m-1}(\tau)$ & $\{0\}$ & $F_{m}(\tau-\varphi)$ & $\{\varphi\}$ \\
\hline$\left(t_{0}+\tau-\varphi\right)^{+}$ & $F_{m-1}(\tau)$ & $\emptyset$ & $F_{m}(\tau-\varphi)$ & $\{\varphi\}$ \\
\hline$t_{0}+\tau$ & $F_{n}\left(F_{m-1}(\tau)+\varphi\right)$ & $\emptyset$ & $F_{n-1}\left(F_{m}(\tau-\varphi)+\varphi\right)$ & $\{0\}$ \\
\hline$\left(t_{0}+\tau\right)^{+}$ & $F_{n}\left(F_{m-1}(\tau)+\varphi\right)$ & $\emptyset$ & $F_{n-1}\left(F_{m}(\tau-\varphi)+\varphi\right)$ & $\emptyset$ \\
\hline
\end{tabular}

Denote $\phi_{1}=F_{n}\left(F_{m-1}(\tau)+\varphi\right)$ and $\phi_{2}=F_{n-1}\left(F_{m}(\tau-\varphi)+\varphi\right)$.

Proposition 2 shows that $\phi_{1}=F_{n}\left(F_{m-1}(\tau)+\varphi\right)>F_{m+n-1}(\tau)>F_{n-1}\left(F_{m}(\tau-\varphi)+\varphi\right)=\phi_{2}$. Then $\varphi_{i}\left(t_{0}+\tau+1-\phi_{1}\right)=1, D_{i}\left(t_{0}+\tau+1-\phi_{1}\right)=\emptyset, \varphi_{j}\left(t_{0}+\tau+1-\phi_{1}\right)=\phi_{2}+1-\phi_{1}$ and $D_{j}\left(t_{0}+\tau+1-\phi_{1}\right)=\emptyset$. Thus, by Lemma 6 , the pulse-coupled oscillator network can not achieve synchronization.

\section{$5 \quad$ Main theorem}

By using the lemmas above, we can obtain our main theorem.

Theorem 1 Under the assumptions (A1) and (A2), from any initial phases $\left(\right.$ other than $\varphi_{1}(0)=$ $\left.\cdots=\varphi_{N}(0)\right)$, the pulse-coupled oscillator network with delayed excitatory coupling can not achieve complete synchronization.

Proof: If $N=2$, then by Lemma 6 the theorem has been proved. Therefore, we only need to consider the case of $N \geq 3$. When $N \geq 3$, for any initial phases (other than $\varphi_{1}(0)=\cdots=\varphi_{N}(0)$ ), 
one of the following two cases must hold.

Case 1: There exists a $t_{1} \geq 0$ such that the network is divided into two cliques (A and B) at time $t_{1}$. That is, the oscillators of clique $\mathrm{A}$ have been synchronized at $t_{1}$ and the oscillators of clique B have also been synchronized at $t_{1}$, but the whole network has not been synchronized at $t_{1}$.

Because the initial phases do not satisfy $\varphi_{1}(0)=\cdots=\varphi_{N}(0)$, there must be a $t_{0}$ with $0 \leq t_{0} \leq t_{1}$ such that the network is divided into two cliques (A and $\mathrm{B}$ ) at time $t_{0}$, and the network is divided into three or more cliques at $t^{\prime}$ for all $0 \leq t^{\prime}<t_{0}$. If $t_{0}=0$, then the network is divided into two cliques at time 0 . By Lemma 6 , it can not achieve synchronization. If $t_{0}>0$, similarly as in the proof of Lemma 5 , there must be an oscillator $i$ satisfying $D_{i}\left(t_{0}\right)=\{0\}$. Then by Lemma $2(\mathrm{c})$, we have $0<\varphi_{i}\left(t_{0}\right)<1$. Since the order of the oscillators is inessential to synchronization, we can re-arrange the order and assume that clique A includes oscillator $1, \ldots, m$ and clique $\mathrm{B}$ includes oscillator $m+1, \ldots, N$, with $1 \leq m<N$. Without loss of generality, we assume that oscillator $i$ is in clique A, i.e., $1 \leq i \leq m$. By Lemma 3 , we have $0<\varphi_{1}\left(t_{0}\right)=\cdots=\varphi_{m}\left(t_{0}\right)<1$ and $D_{1}\left(t_{0}^{+}\right)=\cdots=D_{m}\left(t_{0}^{+}\right)=\emptyset$. For clique B, one of the following two cases must hold.

Subcase 1.1: There does not exist $t^{\prime \prime}<t_{0}$ such that the oscillators of clique B have been synchronized at $t^{\prime \prime}$.

By Lemma $4, D_{i}\left(t_{0}\right)=\{0\}$ shows $D_{j}\left(t_{0}\right) \neq\{0\}$ for all $m+1 \leq j \leq N$. Then by Lemma 5 , we get

$$
\begin{aligned}
& \varphi_{1}\left(t_{0}\right)=\cdots=\varphi_{m}\left(t_{0}\right)<1 \\
& D_{1}\left(t_{0}^{+}\right)=\cdots=D_{m}\left(t_{0}^{+}\right)=\emptyset \\
& \varphi_{m+1}\left(t_{0}\right)=\cdots=\varphi_{N}\left(t_{0}\right)=1 \\
& D_{m+1}\left(t_{0}\right)=\cdots=D_{N}\left(t_{0}\right)=\emptyset .
\end{aligned}
$$

So

$$
\begin{aligned}
& \varphi_{1}\left(t_{0}^{+}\right)=\cdots=\varphi_{m}\left(t_{0}^{+}\right)<1 \\
& D_{1}\left(t_{0}^{+}\right)=\cdots=D_{m}\left(t_{0}^{+}\right)=\emptyset \\
& \varphi_{m+1}\left(t_{0}^{+}\right)=\cdots=\varphi_{N}\left(t_{0}^{+}\right)=0 \\
& D_{m+1}\left(t_{0}^{+}\right)=\cdots=D_{N}\left(t_{0}^{+}\right)=\{\tau\} .
\end{aligned}
$$

Similarly as in the proof of Lemma 6, the network can not achieve synchronization. 
Subcase 1.2: There exists a $t^{\prime \prime}<t_{0}$ such that the oscillators of clique B have been synchronized at $t^{\prime \prime}$.

By Lemma 4 , we have $D_{m+1}\left(t_{0}\right)=\cdots=D_{N}\left(t_{0}\right)$. There are two cases again.

(i) $D_{m+1}\left(t_{0}\right)=\cdots=D_{N}\left(t_{0}\right)=\emptyset$.

Then we have

$$
\begin{aligned}
& \varphi_{1}\left(t_{0}\right)=\cdots=\varphi_{m}\left(t_{0}\right)<1 \\
& D_{1}\left(t_{0}^{+}\right)=\cdots=D_{m}\left(t_{0}^{+}\right)=\emptyset \\
& \varphi_{m+1}\left(t_{0}\right)=\cdots=\varphi_{N}\left(t_{0}\right) \leq 1 \\
& D_{m+1}\left(t_{0}\right)=\cdots=D_{N}\left(t_{0}\right)=\emptyset
\end{aligned}
$$

Denote $\varphi_{1}\left(t_{0}\right)=\cdots=\varphi_{m}\left(t_{0}\right)=\phi_{1}$ and $\varphi_{m+1}\left(t_{0}\right)=\cdots=\varphi_{N}\left(t_{0}\right)=\phi_{2}$. If $\phi_{1}=\phi_{2}$, then

$$
\begin{aligned}
& \varphi_{1}\left(t_{0}\right)=\cdots=\varphi_{m}\left(t_{0}\right)=\varphi_{m+1}\left(t_{0}\right)=\cdots=\varphi_{N}\left(t_{0}\right)=\phi_{1}<1 \\
& D_{1}\left(t_{0}^{+}\right)=\cdots=D_{m}\left(t_{0}^{+}\right)=D_{m+1}\left(t_{0}^{+}\right)=\cdots=D_{N}\left(t_{0}^{+}\right)=\emptyset .
\end{aligned}
$$

By Lemma 3, the network has been synchronized at $t_{0}$, which contradicts that $t_{0} \leq t_{1}$. Thus, $\phi_{1} \neq \phi_{2}$. Without loss of generality, we may assume $\phi_{1}<\phi_{2}$. So, we have

$$
\begin{aligned}
& \varphi_{1}\left(t_{0}+1-\phi_{2}\right)=\cdots=\varphi_{m}\left(t_{0}+1-\phi_{2}\right)=\phi_{1}+1-\phi_{2}<1 \\
& D_{1}\left(t_{0}+1-\phi_{2}\right)=\cdots=D_{m}\left(t_{0}+1-\phi_{2}\right)=\emptyset \\
& \varphi_{m+1}\left(t_{0}+1-\phi_{2}\right)=\cdots=\varphi_{N}\left(t_{0}+1-\phi_{2}\right)=1 \\
& D_{m+1}\left(t_{0}+1-\phi_{2}\right)=\cdots=D_{N}\left(t_{0}+1-\phi_{2}\right)=\emptyset
\end{aligned}
$$

By Lemma 6, the network can not achieve synchronization.

(ii) $D_{m+1}\left(t_{0}\right)=\cdots=D_{N}\left(t_{0}\right)=\{\eta\}\left(D_{i}\left(t_{0}\right)=\{0\}\right.$ shows $\left.0<\eta<\tau\right)$.

Then we have

$$
\begin{aligned}
& \varphi_{1}\left(t_{0}\right)=\cdots=\varphi_{m}\left(t_{0}\right)<1 \\
& D_{1}\left(t_{0}^{+}\right)=\cdots=D_{m}\left(t_{0}^{+}\right)=\emptyset \\
& \varphi_{m+1}\left(t_{0}\right)=\cdots=\varphi_{N}\left(t_{0}\right)<1 \\
& D_{m+1}\left(t_{0}\right)=\cdots=D_{N}\left(t_{0}\right)=\{\eta\}
\end{aligned}
$$

Denote $\varphi_{1}\left(t_{0}\right)=\cdots=\varphi_{m}\left(t_{0}\right)=\phi_{1}$ and $\varphi_{m+1}\left(t_{0}\right)=\cdots=\varphi_{N}\left(t_{0}\right)=\phi_{2}$. It follows from Lemma 1 that $\phi_{2}+\eta<1$. 
If $\phi_{1}+\eta>1$, then $\phi_{1}>\phi_{2}$. It follows that

$$
\begin{aligned}
& \varphi_{1}\left(t_{0}+1-\phi_{1}\right)=\cdots=\varphi_{m}\left(t_{0}+1-\phi_{1}\right)=1 \\
& D_{1}\left(t_{0}+1-\phi_{1}\right)=\cdots=D_{m}\left(t_{0}+1-\phi_{1}\right)=\emptyset \\
& \varphi_{m+1}\left(t_{0}+1-\phi_{1}\right)=\cdots=\varphi_{N}\left(t_{0}+1-\phi_{1}\right)=\phi_{2}+1-\phi_{1}<1 \\
& D_{m+1}\left(t_{0}+1-\phi_{1}\right)=\cdots=D_{N}\left(t_{0}+1-\phi_{1}\right)=\left\{\eta-\left(1-\phi_{1}\right)\right\}
\end{aligned}
$$

where $0<\eta-\left(1-\phi_{1}\right)<\tau$. By Lemma 7 , the network can not achieve synchronization.

If $\phi_{1}+\eta \leq 1$, then $\phi_{1} \leq \phi_{2}$. It follows that

$$
\begin{aligned}
& \varphi_{1}\left(t_{0}+\eta\right)=\cdots=\varphi_{m}\left(t_{0}+\eta\right)=F_{N-m}\left(\phi_{1}+\eta\right) \\
& D_{1}\left(t_{0}+\eta\right)=\cdots=D_{m}\left(t_{0}+\eta\right)=\emptyset \\
& \varphi_{m+1}\left(t_{0}+\eta\right)=\cdots=\varphi_{N}\left(t_{0}+\eta\right)=F_{N-m-1}\left(\phi_{2}+\eta\right) \\
& D_{m+1}\left(t_{0}+\eta\right)=\cdots=D_{N}\left(t_{0}+\eta\right)=\{0\} .
\end{aligned}
$$

Let $K$ be a set of oscillators: oscillator $k^{\prime} \in K$ if and only if $D_{k^{\prime}}\left(t_{0}\right)=\{0\}$. And let $n$ be the number of the oscillators in $K$. It is obvious that clique $\mathrm{B} \cap K=\emptyset$ and clique $\mathrm{A} \supseteq K$. From Proposition 1 and $\phi_{1}, \phi_{2}<1$, it follows that $\phi_{1}=\varphi_{i}\left(t_{0}\right)=f^{-1}\left(f\left(\varphi_{i}\left(t_{0}^{-}\right)\right)+(n-1) \varepsilon\right)$ and $\phi_{2}=\varphi_{m+1}\left(t_{0}\right)=f^{-1}\left(f\left(\varphi_{m+1}\left(t_{0}^{-}\right)\right)+n \varepsilon\right)$. Since $D_{i}\left(t_{0}\right)=\{0\}$ and $D_{m+1}\left(t_{0}\right)=\{\eta\}(0<$ $\eta<\tau)$, by Lemma $2(\mathrm{~d})$, we have $\varphi_{i}\left(t_{0}^{-}\right)>\varphi_{m+1}\left(t_{0}^{-}\right)$. So, $f\left(\phi_{2}\right)-f\left(\phi_{1}\right)=\left[f\left(\varphi_{m+1}\left(t_{0}^{-}\right)\right)+\right.$ $n \varepsilon]-\left[f\left(\varphi_{i}\left(t_{0}^{-}\right)\right)+(n-1) \varepsilon\right]<\varepsilon$. From the concavity assumption of function $f$, it follows that $f\left(\phi_{2}+\eta\right)-f\left(\phi_{1}+\eta\right)<\varepsilon$. Thus, $F_{N-m}\left(\phi_{1}+\eta\right) \neq F_{N-m-1}\left(\phi_{2}+\eta\right)$. Then, similarly as in the proof of Lemma 6 , the network can not achieve synchronization.

Case 2: There does not exist $t_{1} \geq 0$ such that the network is divided into two cliques at time $t_{1}$.

Assume that the pulse-coupled network can achieve synchronization.

Then there must be a $t_{0}>0$ such that the network has been synchronized at $t_{0}$, but the network has not been synchronized at $t$ for all $0 \leq t<t_{0}$. Similarly as in the proof of Lemma 5 , there must be an oscillator $i$ such that $D_{i}\left(t_{0}\right)=\{0\}$. Because there does not exist $t_{1} \geq 0$ such that the network is divided into two cliques at time $t_{1}$, there must be two other oscillators $j$ and $k$ such that

oscillator $i$ and $j$ have not been synchronized at $t$ for all $t<t_{0}$; oscillator $j$ and $k$ have not been synchronized at $t$ for all $t<t_{0}$; oscillator $k$ and $i$ have not been synchronized at $t$ for all $t<t_{0}$. 
By Lemma $4, D_{i}\left(t_{0}\right)=\{0\}$ shows that $D_{j}\left(t_{0}\right) \neq\{0\}$ and $D_{k}\left(t_{0}\right) \neq\{0\}$. So, by Lemma 5 , we have

$$
\begin{aligned}
& \varphi_{j}\left(t_{0}^{-}\right) \neq \varphi_{k}\left(t_{0}^{-}\right) \\
& \varphi_{j}\left(t_{0}\right)=\varphi_{k}\left(t_{0}\right)=1 \\
& D_{j}\left(t_{0}\right)=D_{k}\left(t_{0}\right)=\emptyset
\end{aligned}
$$

However, $\varphi_{i}\left(t_{0}\right)=\varphi_{j}\left(t_{0}\right)=\varphi_{k}\left(t_{0}\right)=1$ contradicts $D_{i}\left(t_{0}\right)=\{0\}$ by Lemma $2(\mathrm{c})$.

So, in this case, the network can not achieve synchronization too.

In summary, the theorem is proved.

Theorem 1 shows that the presence of transmission delays can lead to desynchronization. However, one would say that the definition of complete synchronization in the theorem is awfully strong: it requires that all oscillators reach perfect synchronization in finite time; and say that usually synchronization only requires that the phase differences between oscillators converge to zero as time goes to infinity, i.e., asymptotical synchronization. In Mirollo and Strogatz's model this distinction is irrelevant, since if the phase differences are sufficiently small, perfect synchronization will be achieved after the next round of firings. But this is no longer the case when there exist transmission delays. Large numbers of numerical examples show that when there exist transmission delays, the phase differences can be small but there could still be big variations in the times to process the pulses in the pipeline. Simulation 1 in the following gives an example. Basing on the numerical result, we conjecture that asymptotical synchronization also cannot be achieved in pulse-coupled oscillator networks with delayed excitatory coupling.

If our conjecture is true, then another problem arises: since neither complete synchronization nor asymptotical synchronization can be achieved, what does the model do? We numerically found that the model usually converges to two or more phased-locked clusters. It may be used to explain the phenomenon of clustering synchronization in some species of fireflies, which follow the phase advance model [2]. Simulation 2 in the following shows this.

\section{Numerical simulations}

In this section, we give two numerical simulations. 
Consider the pulse-coupled oscillator network with delayed excitatory coupling:

$$
\begin{aligned}
& f(\varphi)=-I \exp \left\{\ln \frac{I-1}{I} \cdot \varphi\right\}+I \quad(I=1.05) \\
& N=100 \\
& \varepsilon=0.001 \\
& \tau=0.1
\end{aligned}
$$

Obviously, it satisfies all the conditions of Theorem 1. In particular, we have $f(2 \tau)+N \varepsilon=$ $0.5789<1$. So, we conclude that the network can not achieve complete synchronization.

\section{Simulation 1}

In this simulation, we illustrate that even if all the initial phases are very close to each other, there could still be big variations in the times to process the pulses in the pipeline. An example with the initial phases chosen from a uniform distribution on $(0,0.01]$ is plotted in Fig.4.

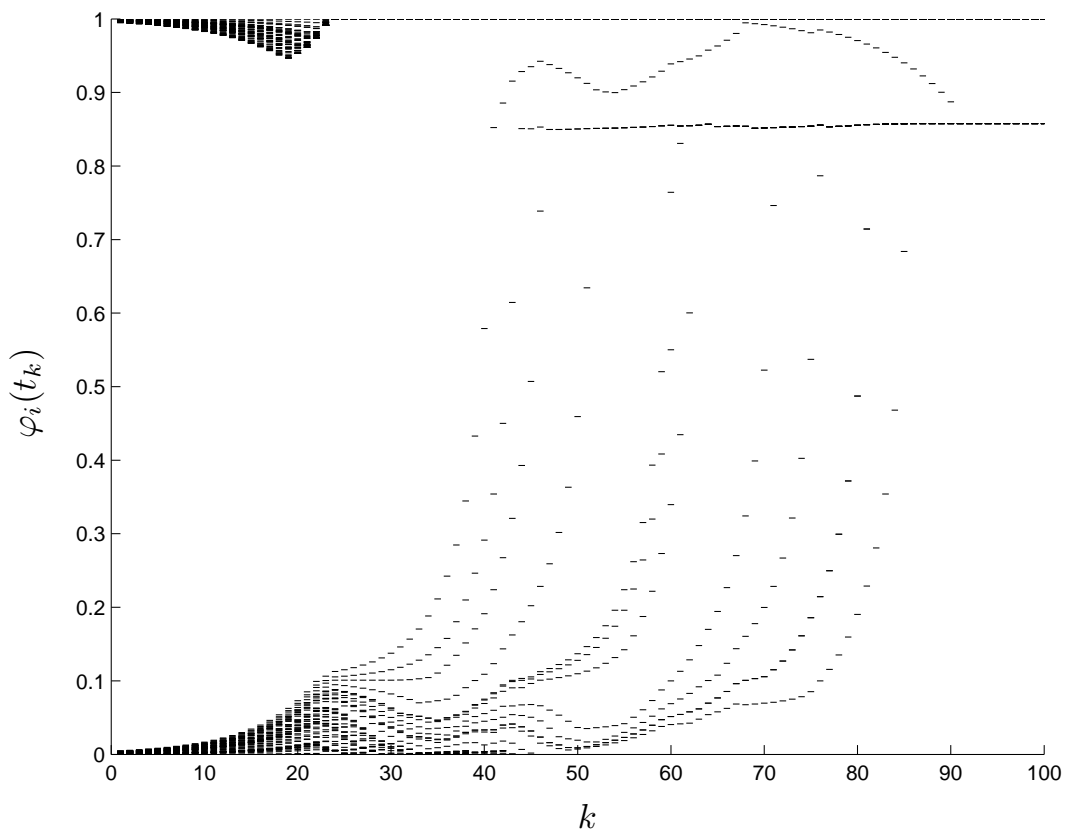

Fig.4: Stroboscopic view on the phases $\varphi_{i}\left(t_{k}\right)$ of $N=100$ oscillators plotted each time $t_{k}$ the first oscillator fires its $k$ th time, i.e., $\varphi_{1}\left(t_{k}\right)=1$. The initial phases are chosen from a uniform distribution on $(0,0.01]$. 


\section{Simulation 2}

In this simulation, we investigate the clustering phenomena. The oscillators are initialized with a uniform random distribution on $(0,1]$. Fig.5 shows that the network can converge to two or more phased-locked clusters.

(a)

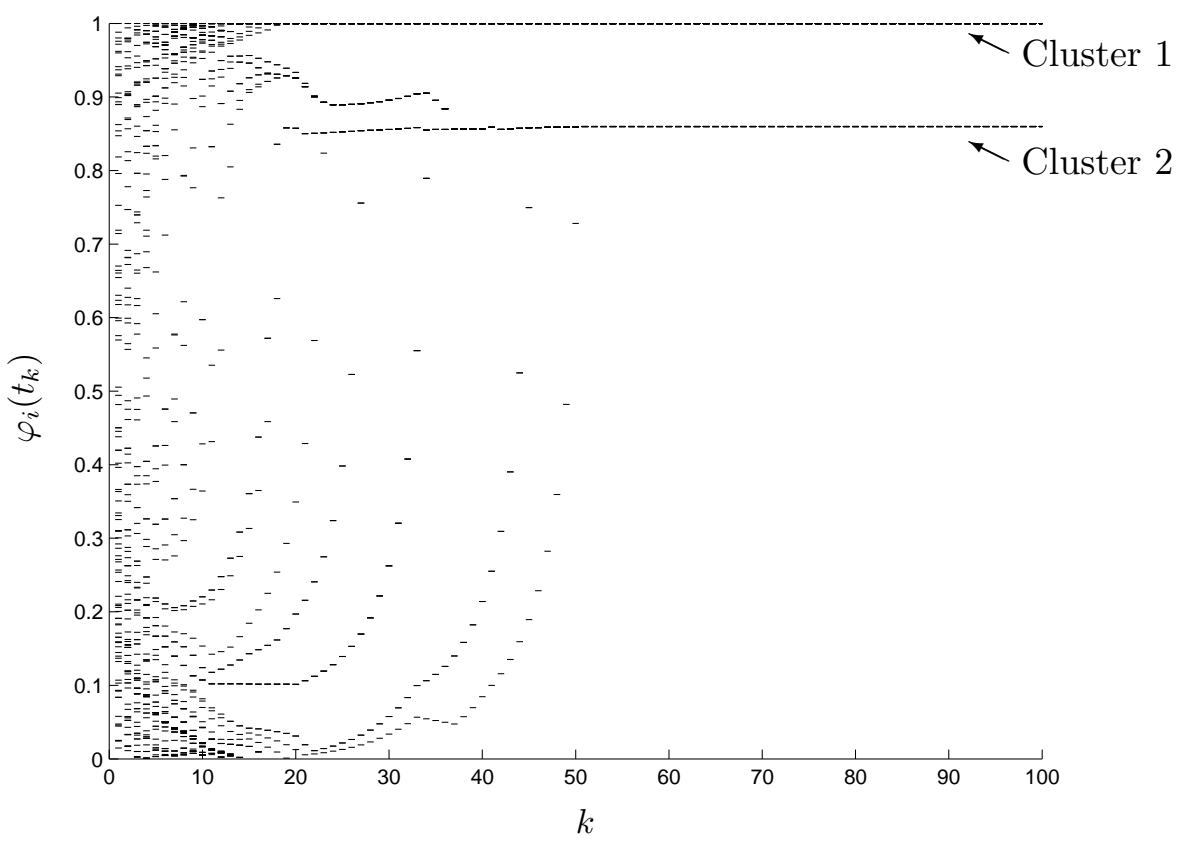

(b)

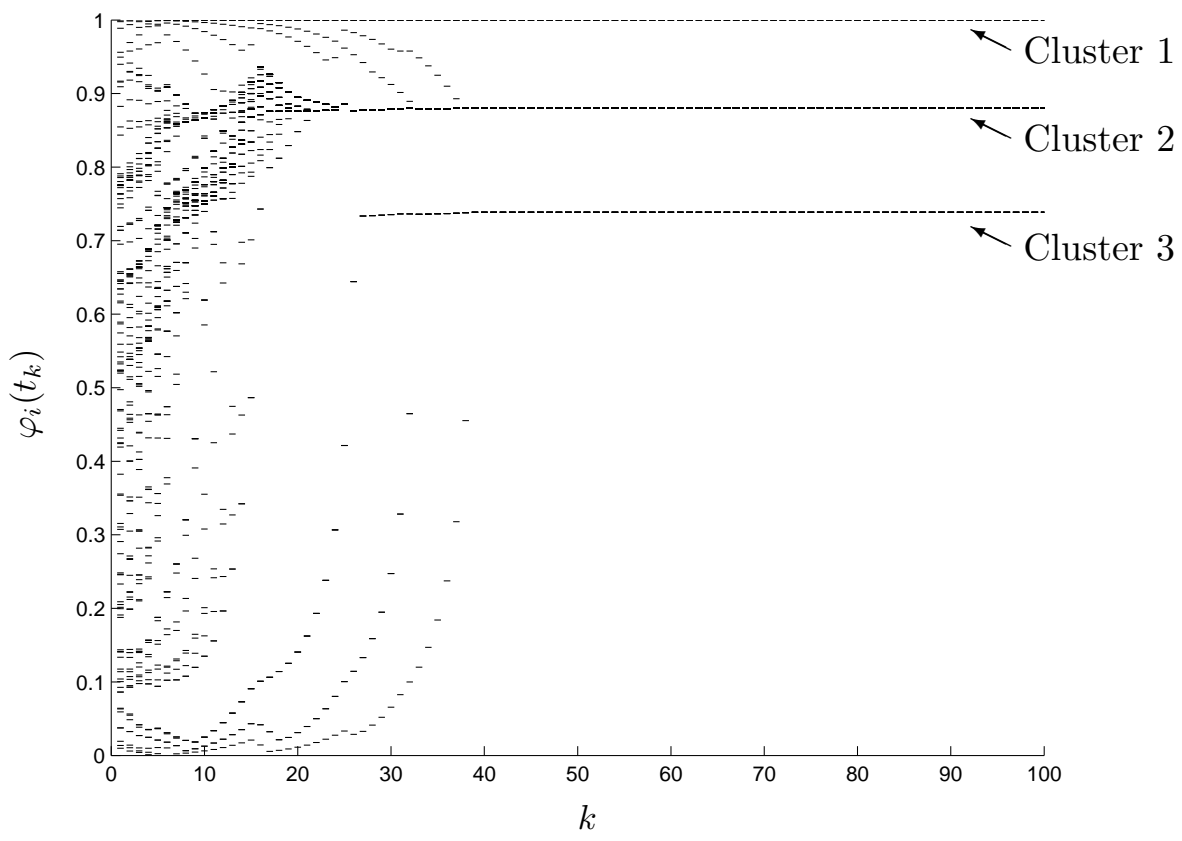

Fig.5: Stroboscopic view on the phases $\varphi_{i}\left(t_{k}\right)$ of $N=100$ oscillators plotted each time $t_{k}$ the first oscillator fires its $k$ th time, i.e., $\varphi_{1}\left(t_{k}\right)=1$. The initial phases are chosen from a uniform distribution on $(0,1]$. Eventually, the network is divided into two or more phased-locked clusters, for example, (a) two clusters (b) three clusters. 


\section{Conclusions}

In this paper, pulse-coupled oscillator networks with delayed excitatory coupling are studied. We propose an assumption (A2), which is reasonable to real biological systems, especially to fireflies. It is proved that under the assumptions (A1) and (A2), from any initial phases (other than $\left.\varphi_{1}(0)=\cdots=\varphi_{N}(0)\right)$, the network can not achieve complete synchronization. This result can explain why Photinus pyralis rarely synchronizes flashing, which is known as an example of pulse-coupled oscillator networks with delayed excitatory coupling. Furthermore, according to Simulation 1, we conjecture that asymptotical synchronization also cannot be achieved; and in Simulation 2, we exhibit a phenomenon of clustering synchronization.

\section{References}

[1] J. Buck, E. Buck, Synchronous fireflies, Sci. Am. 234 (1976) 74-85.

[2] J. Buck, Synchronous rhythmic flashing of fireflies, Part II. Q. Rev. Biol. 63 (3) (1988) 265-289.

[3] F.E. Hanson, Comparative studies of firefly pacemakers, Fed. Proc., 37 (1978) 2158-2164.

[4] H.M. Smith, Synchronous flashing of fireflies, Science, 82 (1935) 151.

[5] C.S. Peskin, Mathematical aspects of heart physiology, Courant Institute of Mathematical Sciences, New York University, New York, (1975) 268-278.

[6] R.E. Mirollo and S.H. Strogatz, Synchronization of pulse-coupled biological oscillators, SIAM J. Appl. Math., 50 (1990) 1645-1662.

[7] Y. Kuramoto, Collective synchronization of pulse-coupled oscillators and excitable units, Phys. D., 50 (1991) 15-30.

[8] C. Vanvreeswijk, L.F. Abbott, Self-sustained firing in populations of integrate-and-fire neurons, SIAM J. Appl. Math., 53 (1993) 253-264.

[9] P. Goel, B. Ermentrout, Synchrony, stability, and firing patterns in pulse-coupled oscillators, Phys. D., 163 (2002) 191-216. 
[10] C.C. Chen, Threshold effects on synchronization of pulse-coupled oscillators, Phys. Rev. E, 49 (1994) 2668-2672.

[11] A. Corral, C.J. Perez, A. Diazguilera, et al, Self-organized criticality and synchronization in a lattice model of integrate-and-fire oscillators, Phys. Rev. Lett., 74 (1995) 118-121.

[12] R. Mathar, J. Mattfeldt, Pulse-coupled decentral Synchronization, SIAM J. Appl. Math., 56 (1996) 1094-1106.

[13] A. Nischwitz, H. Glunder, "Local lateral inhibition-a key to spike synchronization", Biol. Cybern., 73 (1995) 389-400.

[14] U. Ernst, K. Pawelzik, T. Geisel, Synchronization induced by temporal delays in pulsecoupled oscillators, Phys. Rev. Lett., 74 (1995) 1570-1573.

[15] U. Ernst, K. Pawelzik, T. Geisel, Delay-induced multistable synchronization of biological oscillators, Phys. Rev. E., 57 (1998) 2150-2162.

[16] A. Knoblauch, G. Palm, "Scene segmentation by spike synchronization in reciprocally connected visual areas. II. Global assemblies and synchronization on larger space and time scales", Biol. Cybern., 87 (2002) 168-184.

[17] S. Coombes, G.J. Lord, Desynchronization of pulse-coupled integrate-and-fire neurons, Phys. Rev. E., 55 (1997) 2104-2107.

[18] D.E. Kim, A spiking neuron model for synchronous flashing of fireflies, Biosystems, 76 (2004) 7-20. 